\title{
Some Theoretical Links Between Shortest Path Filters and Minimum Spanning Tree Filters
}

\author{
Sravan Danda* · Aditya Challa* • B S Daya Sagar* • Laurent Najman ${ }^{\dagger}$
}

Received: date / Accepted: date

\begin{abstract}
Edge-aware filtering is an important pre-pro cessing step in many computer vision applications. In literature, there exist several versions of collaborative edge-aware filters based on spanning trees and shortest path heuristics which work well in practice. For instance, Tree Filter (TF) which is recently proposed based on a minimum spanning tree (MST) heuristic yields promising results in many filtering applications. However, links between the tree-based filters and shortest path-based filters are faintly explored. In this article, we introduce an edge-aware generalization of the TF, termed as UMST filter based on a subgraph generated by edges of all MSTs. The major contribution of this paper is establishing theoretical links between filters based on MSTs and filters based on geodesics via power watershed framework. More precisely, we show that Union of Minimum Spanning Trees (UMST) filter can be obtained as the limit of Shortest Path Filters (SPFs). Intuitively, TF can be viewed as an approximate limit of the SPFs. We propose and provide a detailed analysis of two different implementations of the UMST filter based on shortest paths. Further, we establish empirically with the help of denoising experiments
\end{abstract}

A preliminary version is published as 'Power tree filter: A theoretical framework linking shortest path filters and minimum spanning tree filters' in International Symposium on Mathematical Morphology and Its Applications to Signal and Image Processing, Springer, 2017, pp 199-210.

Sravan Danda

E-mail: sravan8809@gmail.com, sravandanda@isibang.ac.in. +919663599288

* Systems Science and Informatics Unit, Indian Statistical Institute, Bengaluru, India . † Université Paris-Est, Laboratoire d'Informatique Gaspard-Monge, Équipe A3SI, ESIEE Paris, France that $\mathrm{TF}$ is an approximate limit by showing that $\mathrm{TF}$ and one of our approximations yield similar results.

Keywords Optimization · Image Filtering · Power Watershed $\cdot$ MST $\cdot$ Shortest Paths

\section{Introduction}

Image filtering has been a fundamental problem in computer vision for several years. Edge-preserving filtering is a crucial step in many low-level vision problems such as image denoising [32], image abstraction [32], texture removal [32], texture editing [32], scene simplification [32], stereo matching [32], optical flow [32] etc. These applications are similar in nature and the desired properties of a filter can thus be explained using a denoising application: real world images often contain noise and irrelevant information such as texture along with the object boundaries (which are the major image structures). Hence the goal of an image filtering algorithm is to preserve the object boundaries while getting rid of the redundant information.

In the literature, there exist several edge-aware smoothing filters such as bilateral filter (BF) [33], guided filter (GF) [24], weighted least squares filter (WLS) [20], $L_{0}$ smoothing [37], propagated image filter [10], morphological amoebas or adaptive kernel based filters [25], tree filter $(\mathrm{TF})$ [5] and relative total variation filter (RTV) [38] etc. Specifically, collaborative edge-aware filters based on spanning tree heuristics $[31,5]$ and shortest path heuristics [25] work well in practice but their links are not explored.

In this article, we study the recent edge-aware Tree Filter (TF) [5] which is based on a Minimum Spanning Tree (MST) heuristic. TF admits a linear time algorithm [40] and yields promising results in applications 
such as denoising, texture removal, stereo matching and scene simplification. However it exhibits a leak problem at some of the object boundaries. This problem occurs due to the presence of some object boundary edges in the MST and cannot be avoided as any spanning tree connects all the nodes in a connected graph. Although, the authors in [5] tried to negate the leak effect using a bilateral filter as a post-processing step, the problem still persists (see Fig 1(c)). Also, the filtering results vary with the choice of MST which is undesirable.

This motivated us to explore the links between the $\mathrm{TF}$ and the other edge-aware filtering methods. Further, such links might provide a possibility to design novel edge-aware filters. For example, one can use these links to construct edge-aware features to design supervised edge-aware filters. The main theme of this article is to identify such links and we establish a bridge between filters based on shortest paths and those based on minimum spanning trees. This paper is an extended version of the conference paper [17], our contributions are the following:

1. We introduce an edge-aware filter based on a subgraph generated by union of edges of all MSTs, UMST filter, a generalization of the TF which is an established filtering tool.

2. We show that the UMST filter (see Sec 4 for details) is the limit of SPFs. Intuitively, TF can be visualised as an approximate limit of the SPFs.

3. We propose two different implementations of the UMST filter that serve as alternatives to the TF (see Sec 5 for details) with a detailed analysis of their merits and demerits relative to TF.

4. We establish empirically with the help of denoising experiments that $\mathrm{TF}$ is an approximate limit by showing that TF and one of our approximations yield similar results.

We remark that in the conference version, we have introduced the UMST filter and have stated that it is the limit of SPFs without providing a proof. Also, in the conference article, we have only provided one of the two alternate approximations to TF with some preliminary comparisons between our implementation and TF.

The rest of the paper is organized as follows: In Sec 2 , we briefly recall the notions of $\mathrm{TF}$, power watershed (PW) framework and justify the need for a filter based on a subgraph generated by union of edges of all MSTs (UMST). In Sec 3, we develop SPFs as edge-aware filters starting from Gaussian-like filters and discuss their properties, links with other geodesic based methods. In Sec 4, we show that the UMST filter is the limit of the SPFs in the PW framework. In Sec 5, we discuss approximations of UMST filter i.e. TF and propose two approximations based on shortest paths. We provide a detailed analysis of each of these implementations and empirically establish with the help of denoising experiments that $\mathrm{TF}$ and one of our approximations yield similar results. In Sec 7, the conclusions follow and we speculate some possible directions to extend the ideas in the paper.

\section{From Tree Filter to Union Minimum Spanning Tree Filter}

In this section, we briefly recall the Tree Filter (TF) and provide our motivation on why one should consider using a filter based on a subgraph generated by union of edges of all MSTs (UMST). We then briefly discuss the PW framework and mention some of it's recent applications.

\subsection{Tree Filter}

Suppose $I$ is a given image which possibly contains noise, we let $I_{i}$ denote the color or intensity of the pixel $i$ in the image $I$. The authors in [5] construct a 4-adjacency edge-weighted graph, with the weights between adjacent pixels reflecting the color or intensity dissimilarity. More formally, if $i$ and $j$ are 4-adjacent pixels, they use $w_{i j}$ defined by

$w_{i j}=\left\|I_{i}-I_{j}\right\|_{1}$.

One can construct an MST on this edge-weighted graph, $I_{M S T}$. Since a spanning tree connects every pair of pixels and does not contain cycles, there exists a unique path between every pair of pixels. Let $D(i, j)$ denote the number of edges on the path between $i$ and $j$ on $I_{M S T}$. We also use $I$ to denote the vertex set of this graph i.e. the set of pixels whenever there is no ambiguity. For each pair $i$ and $j$, the collaborative weights $t_{i}(j)$ are given by:

$t_{i}(j)=\frac{\exp \left(\frac{-D(i, j)}{\sigma}\right)}{\sum_{q \in I} \exp \left(\frac{-D(i, q)}{\sigma}\right)}$,

where $\sigma$ controls the falling rate. Intuitively, a high value of $\sigma$ leads to an unweighted average and thus a blurry filter. On the other hand, a very low value of $\sigma$ results in no practical effect of filtering.

The tree filtered value at pixel $i$ is given by

$T F_{i}=\sum_{j \in I} t_{i}(j) I_{j}$ 


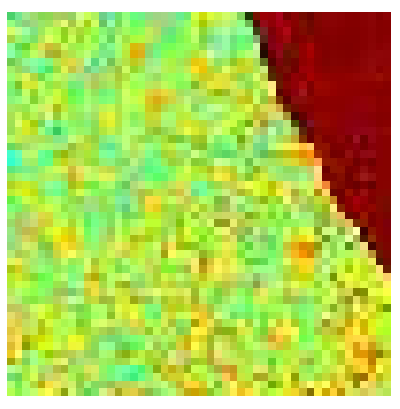

(a)

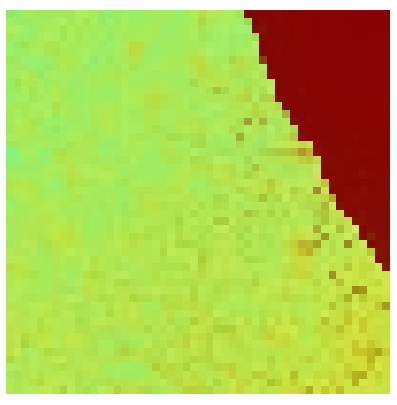

(b)

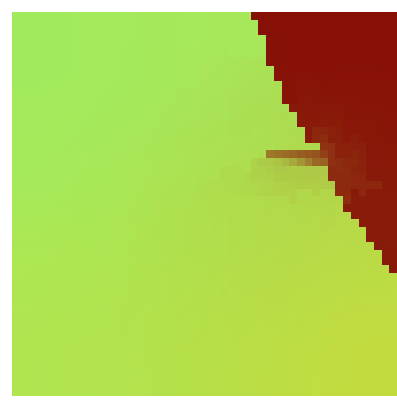

(c)

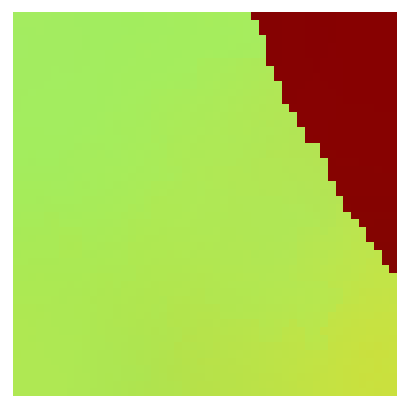

(d)

Fig. 1 (a) Original image (b) Bilateral filter $(\sigma$ color $=100, \sigma$ space $=10)($ c) Tree filter $(\sigma=10)+$ Bilateral filter $(\sigma$ color $=100, \sigma$ space $=10)(\mathrm{d})$ Power Tree Filter $(\sigma=10, N=100)+$ Bilateral filter $(\sigma$ color $=100, \sigma$ space $=10)$.

It is reasonable to assume that the pixel color or intensities vary vastly across objects and are similar within objects. In other words, the higher weight edges mostly correspond to object boundaries and lower weight edges mostly correspond to object interiors. We shall work under this assumption in the rest of the article. The TF works on the following intuition: most of the higher weight edges do not appear while the lower weight edges mostly do appear in any MST. The collaboration across object boundaries is thus low while smoothing within objects is achieved well.

\subsection{Why UMST filter?}

The edges in the image graph that do not belong to object boundaries induce a disconnected subgraph. On the other hand, an MST of a graph is connected, hence any MST contains one or more object boundary edges. These edges cause a leak effect in the tree filtered image (see Fig 1(c)). Also, the filtering results vary with the MST used, making the choice of an arbitrary MST debatable. On the other hand, a filter based on UMST would ensure the following:

1. The filtering results would not depend on arbitrary MST computations.

2. There would be a significant reduction in the leak effect when compared to the TF (see Fig 1(d) for an illustration).

The first property is a direct consequence of the fact that UMST filter uses all the MSTs of the image graph. The second property can be explained intuitively as follows: The edges in the UMST is a superset of the edges of an arbitrary MST. Now, among the edges that belong to UMST but not the MST are mostly object interior edges (compare Fig 2(c) with Fig 2(b)). These object interior edges dominate the collaborative effect of the object boundary edges to ensure a reduction in the leakage. Fig 2 illustrates the above properties on a synthetic image.

Extending the idea of TF, one can use an exponential falling weight similar to Eq 2 for computing collaborative weights. However, we observe that there are possibly multiple paths between a given pair of pixels $i$ and $j$ in the UMST unlike in an MST. In order to define the collaborative weights of the UMST filter, we need a criterion to choose a path among all the paths between $i$ and $j$ in the UMST. Among all paths between $i$ and $j$ in the UMST, loosely speaking, a criterion is to discard paths with 'bad' histograms of the edge weights. More precisely, let $w_{1}<\cdots<w_{k}$ be the distinct weights in the graph and let $P$ and $Q$ be paths between $i$ and $j$ in the UMST with $P$ having $m_{1}$ edges of weight $w_{1}, \cdots, m_{k}$ edges of weight $w_{k}$ and $Q$ having $n_{1}$ edges of weight $w_{1}, \cdots, n_{k}$ edges of weight $w_{k}$. We compare the histograms of the edge-weights i.e. $\left(m_{1}, \cdots, m_{k}\right)$ and $\left(n_{1}, \cdots, n_{k}\right)$ as follows: if $m_{k}>n_{k}$ or if $m_{r}=n_{r}, l<r \leq k$ along with $m_{l}>n_{l}$, then we discard path $P$. Essentially, we pick a path that is intuitively consistent with the assumption that pixel color or intensities vary vastly across objects and are similar within objects. The reader may refer to Sec 4 for formal definitions and properties.

Now, we shall recall notions of the power watershed framework and a few of it's recent applications.

\subsection{Power Watershed Framework and Related Work}

The study of asymptotic behaviour of minimizers has been useful in many computer vision applications especially the ones that are based on total variation formulation. The most popular approach is to use the notion of $\Gamma$-convergence [7] where one computes the limit of minimizers of simpler minimization problems to approximate the solution to a complex minimization problem under a sufficient set of conditions. There are nu- 


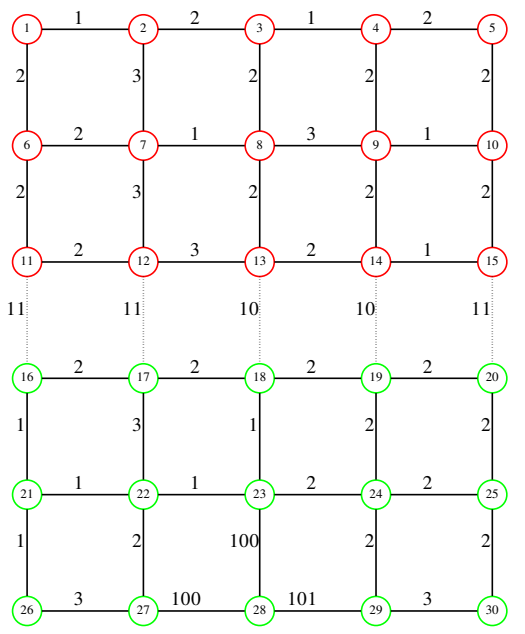

(a)

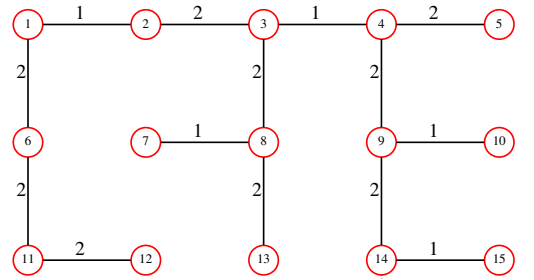

10

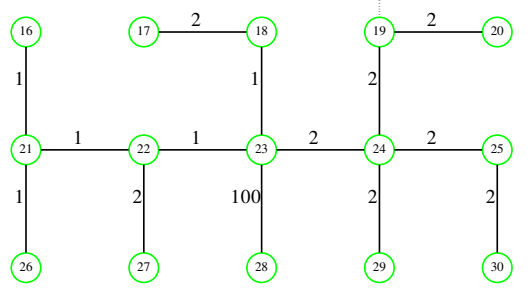

(b)
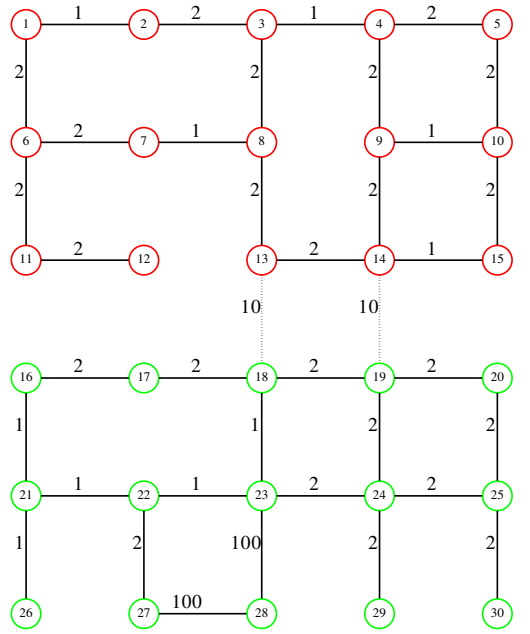

(c)

Fig. 2 (a) 4-adjacency graph of a synthetic image containing two objects coloured in red and green. The pixels in this image are indexed from 1 to 30 and the weights on the edges denote the intensity dissimilarities. The edges corresponding to object boundaries are represented by dotted lines, (b) An MST obtained from (a), and (c) UMST obtained from (a). In order to illustrate that UMST filter yields better results, it should perform at least as good as TF for - removing noise at pixel numbered 28 and reducing the leak at object boundaries say at pixel numbered 13 and 14. Consider pixel numbered 28. One can see that both the edges of weights 100 incident on this pixel are present in the UMST, the noise removal is enhanced due to higher collaboration with the neighbouring pixels when compared to that of tree filter where MST had only one of the edges with weight 100. Now consider the pixel numbered 13. We see that although an extra boundary edge (edge 13 - 18) appears in the UMST, the presence of an additional interior edge incident on 13 in the UMST ensures that the effect of the boundary edge collaboration is reduced significantly. At pixel numbered 14, the UMST filter performs better than tree filter due the presence of the additional interior edge 13 - 14. Similar arguments hold for pixels numbered 18 and 19.

merous applications in computer vision that fall in this category. However, there are other similar notions of investigating limits of solutions to minimization problems that have been proved to be useful. For instance, in $[28,2]$, the elementary mathematical morphological (MM) operators have been formulated as limits of variational problems; and in [34], the authors view the local min-max filters as a limit of normalized power-weighted averaging filter.

Power Watershed (PW) framework which is a closely related notion to $\Gamma$-convergence investigates limits of solutions of minimization problems on finite edge-weighted graphs by letting the powers of edge-weights tend to $\infty$. Some useful applications include total variation and spectral clustering: In [13], the power watershed framework unifies and extends a common framework of semisupervised or seeded graph-based image segmentation methods namely graph cuts [6], random walker [22], geodesics $[1,4,16,19,30]$ and watershed cuts $[14,15]$; In [9], the authors propose a fast alternative to spectral clustering methods $[36,41]$ by computing their limit. More recently, [27] generalizes the power watershed framework for a larger class of cost functions (Eqns. 1 and 2 in [27]) and formalizes the notion of scale for investigating minimizers. In simple words, [27] develops theory and algorithms to combine data reduction and optimization techniques. As edge-preserving image filtering and image segmentation are closely related problems, one can anticipate to establish links between existing filtering methods using the PW framework $[13,27]$.

In this article, we model the images with finite edgeweighted graphs and show that UMST filter is the limit of SPFs by letting the powers of the edge-weights tend to $\infty$. This result implies that one can view the UMST filter and the shortest path filters in an optimization framework. Although both [27] and this article involve the computation of limit of minimizers of parametrized cost functions, it is important to note that the cost functions used in this article do not fall in the framework of Eqns. 1 and 2 in [27]. The reader may refer to Sec 4 for a proof of the main result.

\section{Shortest Path Filters and Related Methods}

In this section, we shall review the shortest path filters in detail. In particular, we develop them as a natural edge-aware extension of Gaussian-like filters. The rest of the section is dedicated to the discussions on their links with other related geodesic methods.

Before formally defining the SPF, we need some notions of graphs specific to modelling images: 


\subsection{Basic Notions}

Definition 1 An edge-weighted graph $\mathcal{G}=(V, E, W)$ consists of a finite set $V$ of nodes, and set of unordered pairs of elements of $V$ i.e. $\{\{i, j\} \subset V: i \neq j\}$, called the edge set $E$, a positive real-valued function $W$ on the set $E$. We denote $w_{i j}$ or $W\left(e_{i j}\right)$ as the weight of the edge joining pixels $i$ and $j$.

Definition 2 For $p \in \mathbb{Z}^{+}, \mathcal{G}^{(p)}=\left(V, E, W^{(p)}\right)$ is the graph that contains the same set of nodes and edges as of $\mathcal{G}$ and $W^{(p)}\left(e_{i j}\right)=\left(W\left(e_{i j}\right)\right)^{p}$ for each edge $e_{i j} \in E$ and we call $\mathcal{G}^{(p)}$ as an exponentiated graph of $\mathcal{G}$.

Definition 3 A path $P(i, j)$ between nodes $i$ and $j$ is a finite ordered sequence of nodes of $\mathcal{G}$ such that there is an edge incident on every adjacent pair of nodes in the sequence. We say that a path from $i$ to $j$ is a simple path if all the nodes in the sequence are distinct.

Definition 4 An edge-weighted graph $\mathcal{G}=(V, E, W)$ is said to be connected if there exists a path between every pair of nodes in $V$.

In the rest of the article, we only deal with connected graphs and mostly 2 dimensional grid graphs.

Definition 5 Assume that the distinct weights in $\mathcal{G}$ are given by $0<w_{1}<w_{2}<\cdots<w_{k}$. Given a path $P(i, j)$ in $\mathcal{G}$, one can assign a $k$-tuple $\left(n_{1}, \cdots, n_{k}\right)$ to the path, where $n_{r}$ denotes the number of edges of weight $w_{r}$ on the path $P(i, j)$. This $k$-tuple is referred to as the edge-weight distribution of the path $P(i, j)$.

In simple words, edge-weight distribution is a histogram of the edge-weights present in the path. We remark that the $k$-tuples associated with a path $P(i, j)$ in graph $\mathcal{G}$ and its exponentiated graph $\mathcal{G}^{(p)}$ are identical by the virtue of it's definition. However it is important to note that: corresponding to each of the coordinates in the edge-weight distributions, the weights are different.

Definition 6 Assume that the distinct weights in $\mathcal{G}$ are given by $0<w_{1}<w_{2}<\cdots<w_{k}$. Suppose $P(i, j)$ is a path between pixels $i$ and $j$ in $\mathcal{G} . P(i, j)$ is said to be a shortest path between the pixels $i$ and $j$ in $\mathcal{G}$ if for every path $Q(i, j)$ between $i$ and $j$ in $\mathcal{G}$, we have $\sum_{r=1}^{k} n_{r} w_{r} \leq \sum_{r=1}^{k} m_{r} w_{r}$ where $\left(n_{1}, \cdots, n_{k}\right)$ and $\left(m_{1}, \cdots, m_{k}\right)$ denote the edge-weight distributions of $P(i, j)$ and $Q(i, j)$ respectively.

This definition is another way of representing standard shortest path length suited for our purposes. We remark that a shortest path is always a simple path since all the weights in the edge-weighted graphs are strictly positive.
We shall now build an edge-aware filter from scratch: Let $i$ and $j$ be two pixels in $\mathcal{G}^{(p)}$. Consider the simplest weighted-average filter whose collaborative weights are given by

$g_{i}(j)=\frac{\exp \left(-\frac{\|i-j\|_{1}}{\sigma}\right)}{\sum_{k \in V} \exp \left(-\frac{\left.\|i-k\|_{1}\right)}{\sigma}\right)}$,

where $\|i-j\|_{1}: l_{1}$ norm between the pixels $i$ and $j$ and $\sigma$ is the parameter controlling the level of smoothing.

The Gaussian-like filter at pixel $i$ denoted by $G F_{i}$ is given by:

$G F_{i}=\sum_{j \in V} g_{i}(j) I_{j}$.

We observe that the collaborative weights purely depend on the spatial distance between pixels $i$ and $j$ and hence the name Gaussian-like filter. More specifically, collaborative weights between pixels separated by same distance is indifferent w.r.t. existence of an object boundary between them. Hence, one has to find a way to ensure that the collaborative weights are lesser across boundaries. A natural way to extend the idea of a Gaussian-like filter is: given a pair of pixels $i$ and $j$, consider the number of edges on a path with smallest sum of weights between them.

Let $\Pi(P(i, j))$ denote the number of edges on a path $P(i, j)$. Define

$\Theta^{(p)}(i, j)=\inf _{P(i, j)}\{\Pi(P(i, j))\}$

where $P(i, j)$ is a shortest path in $\mathcal{G}^{(p)}$ and the edge weights are given by:

$w_{i j}=\left\|I_{i}-I_{j}\right\|_{1}+1$.

The SPF at pixel $i$ is defined as:

$S P F_{i}^{(p)}=\sum_{j \in V} \frac{\exp \left(-\frac{\Theta^{(p)}(i, j)}{\sigma}\right)}{\sum_{k \in V} \exp \left(-\frac{\Theta^{(p)}(i, k)}{\sigma}\right)} I_{j}$,

where $\sigma$ controls the falling rate.

Note that the weights in Eq 7 are different from the ones in Eq 1. This modification is required so that the edge-weights are all positive. We shall need positive edge-weights to ensure that the shortest paths in limiting case can be nicely characterized (see Lemma 1 


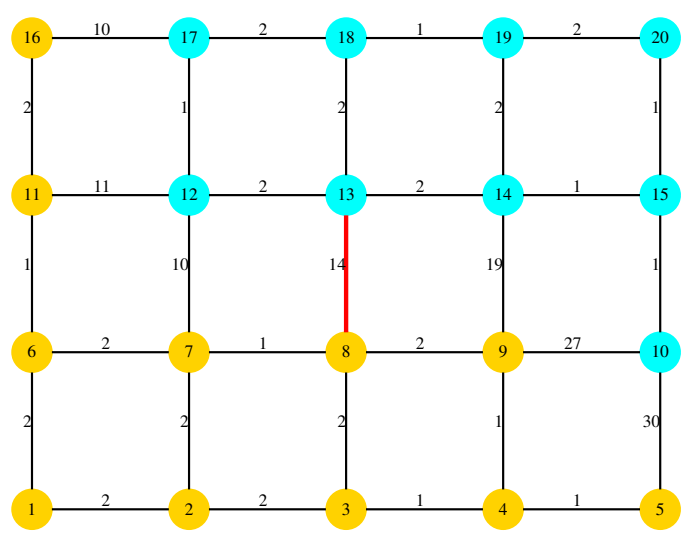

(a)

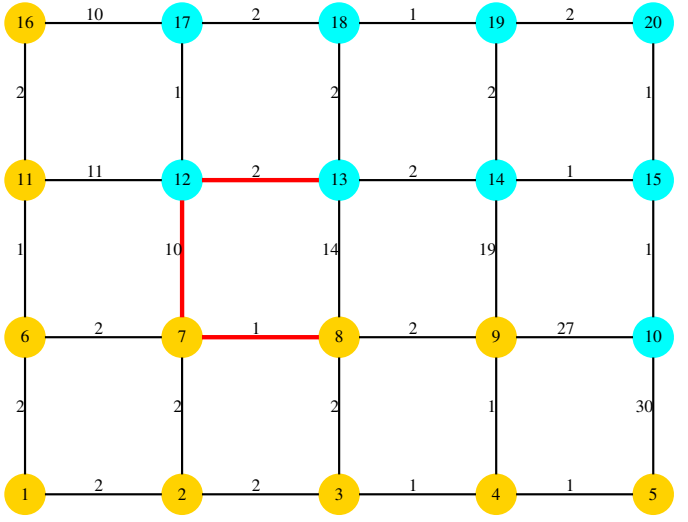

(b)

Fig. 3 (a) and (b) 4-adjacency graph of a synthetic image containing two objects coloured in blue and yellow. The pixels in this image are indexed from 1 to 20 and the weights on the edges denote the intensity dissimilarities. The collaborative weight for the pair of pixels 8 and 13 (which crosses an object boundary) given by Gaussian-like filter would consider the edge highlighted in red in (a) and yields a high value. On the other hand, the SPF considers the path $<8,7,12,13\rangle$ highlighted in red in (b) for computing the corresponding collaborative weight. This illustrates that SPF respects the object boundaries.

for details). Also, TF and UMST filter are both invariant to increasing transformation on the edge-weights. Hence it is fair to relate MST based filters and SPF both using the weights given by Eq 7 . In the rest of the article, we use the edge weights as per Eq 7.

We remark that in the special case of all pixel values being equal in the image, SPF is exactly Gaussianlike filter. Also, in practice, the shortest path distances between a pair of pixels within the objects are close to the spatial distances and are larger than the spatial distances across object boundaries. Fig 3 illustrates on a synthetic image that the SPF is a natural edgepreserving extension of the Gaussian-like filter.

Further, one can see that the SPF value at pixel $i$ is a solution of the following optimization problem:

Consider the cost function

$Q_{i}^{(p)}(x)=\sum_{j \in V} \exp \left(-\frac{\Theta^{(p)}(i, j)}{\sigma}\right)\left\|x-I_{j}\right\|_{2}^{2}$,

where $\sigma$ controls the falling rate. The shortest path filtered value at pixel $i$ is given by the minimizer of $Q_{i}^{(p)}(x)$ i.e.

$S P F_{i}^{(p)}=\arg \min _{x} Q_{i}^{(p)}(x)$.

SPFs are not completely new and there exist in literature, several edge-preserving filters using geodesics such as the ones discussed in [23], the adaptive kernel filter such as morphological amoebas [25].

\subsection{Relation to Morphological Amoebas}

SPFs are also closely related to Morphological Amoebas [25]. Morphological Amoebas are adaptive structuring elements based on shortest path distances used to build edge-aware filters. These kernels work on the assumption that the gradients are low within the objects and high across the object boundaries. In order to ensure that the kernels do not cross the object boundaries, the amoeba distance defined below is used to compute them:

$\kappa(i, j)=\min _{P(i, j)} L_{\lambda}(P(i, j))$,

where $P(i, j)$ is a path between pixels $i$ and $j,<i=$ $x_{0}, x_{1}, \cdots, x_{n}=j>$ and $\lambda \geq 0$ is a user input.

$L_{\lambda}(P(i, j))=\sum_{t=0}^{n-1}\left(1+\lambda\left\|I_{x_{t+1}}-I_{x_{t}}\right\|_{1}\right)$.

The closed ball at pixel $i$ given by $\{j: \kappa(i, j) \leq$ $r\}$ is the kernel used for edge-aware smoothing. The cardinality or the size of the kernel depends on $r$ and is chosen as per the level of smoothing desired.

Proposition $1 \Theta^{(1)}(i, j)$ is a constrained minima of the amoeba kernel path length given by

$$
\begin{array}{r}
\Theta^{(1)}(i, j)=\min L_{0}(P(i, j)) \text { subject to } \\
P(i, j) \in \operatorname{argmin} L_{1}(P(i, j)) .
\end{array}
$$

Further, the family of parameters $\Theta^{(p)}(i, j)$ are given by: 


$$
\begin{gathered}
\Theta^{(p)}(i, j)=\min L_{0}^{p}(P(i, j)) \text { subject to } \\
P(i, j) \in \arg \min L_{1}^{p}(P(i, j)),
\end{gathered}
$$

where

$L_{\lambda}^{p}(P(i, j))=\sum_{t=0}^{n-1}\left(1+\lambda\left\|I_{x_{t+1}}-I_{x_{t}}\right\|_{1}\right)^{p}$.

Proof It readily follows from $\mathrm{Eq} 6, \mathrm{Eq} 7, \mathrm{Eq} 11$ and $\mathrm{Eq}$ 14

Note that the morphological amoeba lengths are a special case of the lengths given by Eq 14 . We can hence view the SPF as a generalization of the notion of morphological amoeba lengths.

\section{UMST Filter as a Limit of Shortest Path Filters}

In this section, we shall formally define the UMST filter and prove that the UMST filter is the limit of SPFs. As the weights of the graphs of the shortest path filters are powers of natural numbers (see Sec 3 ), we use the term Power Tree Filter to denote the limit of Shortest Path Filters. We shall need some definitions before we prove this result.

\subsection{Formal Notion of UMST Filter}

Definition 7 Let $\mathcal{G}=(V, E, W)$ be a connected edgeweighted graph. The Union of Minimum Spanning Trees (UMST) of $\mathcal{G}$ is defined as the weighted subgraph induced by edges of all the minimum spanning trees of $\mathcal{G}$.

We remark that the usage of the term 'Union of Minimum Spanning Trees' (UMST) is justified in Def 7. This is because the union of all edges belonging to some MST of the underlying connected graph induces this subgraph.

Definition 8 Assume that graph $\mathcal{G}$ has $k$ distinct weights given by $0<w_{1}<\cdots<w_{k}$. Let $\left(n_{1}, \cdots, n_{k}\right)$ and $\left(m_{1}, \cdots, m_{k}\right)$ denote the edge-weight distributions of paths $P$ and $Q$ in $\mathcal{G}$ respectively. Let $l=\sup (A)$ where $A=\left\{r: 1 \leq r \leq k, n_{r} \neq m_{r}\right\}$ We define reverse dictionary ordering or reverse lexicographic ordering on the set of paths in $\mathcal{G}$ as follows:

$P \geq Q \Leftrightarrow A=\emptyset$ or $n_{l}>m_{l}$

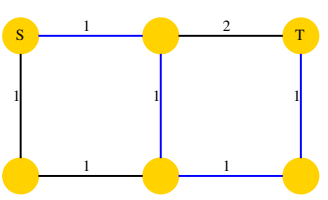

(a)

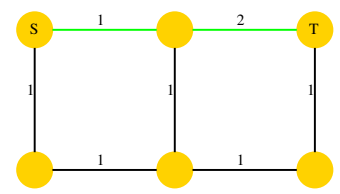

(b)
Fig. 4 (a) and (b) synthetic images to illustrating the reverse lexicographic ordering of paths. The reverse lexicographic order of path in blue is lesser than that of the one in green

See Fig 4 for an illustration on reverse dictionary ordering. Note that reverse dictionary ordering yields a complete ordering on the set of paths in $\mathcal{G}$ and the ordering remains same in each of the exponentiated graphs $\mathcal{G}^{(p)}$.

Definition 9 Suppose $P(i, j)$ is a path between pixels $i$ and $j$ in $\mathcal{G} . P(i, j)$ is said to be a smallest path w.r.t. reverse dictionary order between the pixels $i$ and $j$ in $\mathcal{G}$ if for every path $Q(i, j)$ between $i$ and $j$ in $\mathcal{G}$, we have $Q(i, j) \geq P(i, j)$.

Note that every smallest path w.r.t. reverse dictionary order between pixels $i$ and $j$ in $\mathcal{G}$ has the same edge-weight distribution. In particular, the number of edges on a smallest path w.r.t. reverse dictionary order between $i$ and $j$ denoted by $\Pi(i, j)$ is well-defined.

Any MST in $\mathcal{G}^{(p)}$ is an MST in $\mathcal{G}$ and vice-versa. This follows directly from the fact that MST is invariant to any strictly increasing transformation on the weights of a connected graph. The notions of smallest paths w.r.t. reverse dictionary order and that of MSTs in $\mathcal{G}^{(p)}$ are hence independent of $p$.

Definition 10 Let $\eta(i, j)$ denote the number of edges on a path with smallest reverse dictionary order between $i$ and $j$ on $\mathcal{G}_{U M S T}$ where $\mathcal{G}_{U M S T}$ is the UMST of the image graph $\mathcal{G}$ with 4-adjacency, edge weights given by Eq 7 . For each pair $i$ and $j$, the collaborative weights $\hat{t}_{i}(j)$ are given by:

$\hat{t}_{i}(j)=\frac{\exp \left(\frac{-\eta(i, j)}{\sigma}\right)}{\sum_{q \in V} \exp \left(\frac{-\eta(i, q)}{\sigma}\right)}$,

where $\sigma$ controls the falling rate.

The UMST filtered value at pixel $i$ is given by

$U M S T_{i}=\sum_{j \in V} \hat{t}_{i}(j) I_{j}$

where $I_{j}$ denotes the intensity of the pixel $j$ in the original image. This is a natural way to generalize the TF since: (a) the lesser the reverse lexicographic order of a path, the lesser the chance of the path crossing an 
object boundary, (b) in the special case of the graph having a unique MST, this filter is exactly the same as TF.

\subsection{Characterizing Limit of Shortest Path Filters}

In this subsection, we characterize the Power Tree Filter or limit of Shortest Path Filters. Firstly, we have the following result:

Lemma 1 Let $\mathcal{G}=(V, E, W)$. For every pair of pixels $i$ and $j$ in $V$, there exists $p_{0} \geq 1$ such that, a path $P(i, j)$ is a shortest path between $i$ and $j$ in $\mathcal{G}^{(p)}$ for all $p \geq p_{0}$ if and only if $P(i, j)$ is a smallest path w.r.t. reverse dictionary order between $i$ and $j$ in $\mathcal{G}$. Further, $p_{0}$ is independent of $i$ and $j$.

Proof Let $\mathcal{G}=(V, E, W)$ and let the distinct weights in $\mathcal{G}$ be given by $w_{1}<\cdots<w_{k}$.

Firstly, we shall show that for a given pair of pixels $i$ and $j$, if $P(i, j)$ is a smallest path w.r.t. reverse dictionary order between $i$ and $j$ in $\mathcal{G}$ then there exists a constant $p_{0}$ such that for each $p \geq p_{0}, P(i, j)$ is a shortest path between $i$ and $j$ in $\mathcal{G}^{(p)}$. Let $P(i, j)$ be a smallest path w.r.t. reverse dictionary order between $i$ and $j$ in $\mathcal{G}$. Let $Q(i, j)$ be an arbitrary simple path between $i$ and $j$. Let $\left(n_{1}, \cdots, n_{k}\right)$ and $\left(m_{1}, \cdots, m_{k}\right)$ denote the edgeweight distributions of paths $P(i, j)$ and $Q(i, j)$ respectively. Let $A(P, Q)=\left\{1 \leq r \leq k: n_{r} \neq m_{r}\right\}$. Suppose $A(P, Q)=\emptyset$ then $\sum_{r=1}^{k} n_{r} w_{r}^{p} \leq \sum_{r=1}^{k} m_{r} w_{r}^{p} \forall p \geq 1$. If $A(P, Q) \neq \emptyset$ then let $l=\sup (A(P, Q))$. We have $m_{l}>n_{l}$ by choice of $P(i, j)$. Also, the difference of the total weights i.e. $\sum_{r=1}^{k} m_{r} w_{r}^{p}-\sum_{r=1}^{k} n_{r} w_{r}^{p}=\mathcal{O}\left(w_{l}^{p}\right)$ with a positive leading coefficient. Hence $\exists p_{Q(i, j)} \geq 1$ such that $\sum_{r=1}^{k} n_{r} w_{r}^{p} \leq \sum_{r=1}^{k} m_{r} w_{r}^{p} \forall p \geq p_{Q(i, j)}$. Now, let $\mathcal{S}_{i j}$ denote the set of all simple paths from $i$ to $j$. Then $\left|\mathcal{S}_{i j}\right|<\infty$. Set $p_{i j}=\sup \left\{p_{Q(i, j)}: Q(i, j) \in\right.$ $\left.\mathcal{S}_{i j}\right\}<\infty$. We note that given any path which is not simple, one can drop the redundant edges to construct a simple path with strictly smaller total weight. It is hence enough to show that the total weight of $P(i, j)$ is lesser than or equal to every simple path between $i$ and $j$ in $\mathcal{G}^{(p)}$ for sufficiently large $p$. As $V$ is finite, setting $p_{0}=\sup \left\{p_{i j}: i, j \in V\right\}$ completes the argument.

Conversely, suppose $P(i, j)$ is NOT a smallest path w.r.t. reverse dictionary ordering between $i$ and $j$, we shall construct a sequence $\left(p_{n}\right)_{n \geq 1}$ converging to $\infty$ such that $P(i, j)$ is not a shortest path between $i$ and $j$ in $\mathcal{G}^{\left(p_{n}\right)}$ for each $n \geq 1$. Since $P(i, j)$ is not a smallest path w.r.t. reverse dictionary ordering between $i$ and $j, \exists a$ path $T(i, j)$ between $i$ and $j$ such that $P(i, j) \geq T(i, j)$ holds but $T(i, j) \geq P(i, j)$ does not hold. Equivalently, if the edge weight distributions of $P(i, j)$ and $T(i, j)$ are given by $\left(n_{1}, \cdots, n_{k}\right)$ and $\left(t_{1}, \cdots, t_{k}\right)$ respectively then $A(P, T) \neq \emptyset$ and $t_{l}<n_{l}$ where $l=\sup (A(P, T))$ and $A(P, T)=\left\{1 \leq r \leq k: n_{r} \neq t_{r}\right\}$. The difference of the total weights i.e. $\sum_{r=1}^{k} t_{r} w_{r}^{p}-\sum_{r=1}^{k} n_{r} w_{r}^{p}=\mathcal{O}\left(w_{l}^{p}\right)$ and has a negative leading coefficient. Thus, $\exists$ a constant $\rho_{i j} \geq 1$ such that for each $p \geq \rho_{i j}, P(i, j)$ is not a shortest path between $i$ and $j$. We set $\left(p_{n}\right)_{n \geq 1}$ by $p_{n}=\rho_{i j}+n-1$ to complete the proof.

Loosely speaking, for a large enough power $p$, on $\mathcal{G}^{(p)}$, between every pair of pixels, a shortest path is a smallest path w.r.t. reverse dictionary order and viceversa. In short, we have the following corollary:

Corollary 1 Let $\Delta(i, j)$ denote the number of edges on a smallest path w.r.t. dictionary order between pixels $i$ and $j$ in $\mathcal{G}$. As $p \rightarrow \infty$, we have $\Theta^{(p)}(i, j) \rightarrow \Delta(i, j)$ for each pair $i$ and $j$.

\subsection{Optimization Framework for UMST Filter}

Recall the definition of UMST filter: Let $\mathcal{G}$ denote the given image, let $\mathcal{G}_{U M S T}$ denote the UMST on the edgeweighted graph constructed from $\mathcal{G}$. Let $\eta(i, j)$ denote the number of edges on a smallest path w.r.t. reverse dictionary order between pixels $i$ and $j$ in $\mathcal{G}_{U M S T}$. Now consider the cost function given by:

$$
\widehat{Q}_{i}(x)=\sum_{j \in V} \exp \left(-\frac{\eta(i, j)}{\sigma}\right)\left\|x-I_{j}\right\|_{2}^{2},
$$

where $\sigma$ controls the falling rate, the UMST filtered value at pixel $i$ is given by the minimizer of $\widehat{Q}_{i}(x)$.

$U M S T_{i}=\arg \min \widehat{Q}_{i}(x)=\sum_{j} \frac{\exp \left(-\frac{\eta(i, j)}{\sigma}\right)}{\sum_{k} \exp \left(-\frac{\eta(i, k)}{\sigma}\right)} I_{j}$

where UMST denotes the UMST filtered image.

Firstly, we need the following:

Definition 11 Let $\mathcal{G}=(V, E, W)$ be a connected edgeweighted graph. A subset $C \subset E$ is said be a cut if the subgraph induced by the edge set $E \backslash C$ is not a connected graph.

Lemma 2 (Cut Property) For any cut $C$ of a connected graph $\mathcal{G}=(V, E, W)$, if the weight of an edge $e, e \in C$ is less than or equal to the weight of every other edge in $C$, then this edge belongs to an MST of the graph $\mathcal{G}=(V, E, W)$. 
Lemma 3 (Cycle Property) For any cycle $C$ in the graph $\mathcal{G}=(V, E, W)$, if the weight of an edge e of $C$ is larger than the individual weights of all other edges of $C$, then this edge cannot belong to an MST.

Before we prove the main result of the paper, we need Lemma 4 which is a modified version of a result stated in [27].

Lemma 4 Let $\mathcal{G}=(V, E, W)$ be an edge-weighted graph. Let $\mathcal{G}_{<w}$ denote the induced subgraph of $\mathcal{G}$ with the vertex set $V$ and all the edges $e_{i j} \in E$ whose weight $w_{i j}<$ $w$. Let $\mathcal{G}_{U M S T}$ denote the UMST of $\mathcal{G}$. Then an edge e with weight $w(e)$ belongs to the $\mathcal{G}_{U M S T}$ if and only if the edge e joins two connected components in $\mathcal{G}_{<w(e)}$.

Proof The proof directly follows from Lemma 2 and Lemma 3.

In simple words, an edge $e$ is in some MST if and only if it connects two different components of the induced subgraph generated by edges of weights lower than that of $e$.

Proposition 2 Every smallest path w.r.t. reverse dictionary order between any two arbitrary nodes in $\mathcal{G}=$ $(V, E, W)$ lies on an $M S T$ of $\mathcal{G}$ and hence on the UMST of $\mathcal{G}$.

Proof Let $i$ and $j$ be two arbitrary nodes in $\mathcal{G}$. Let $P(i, j)$ be a smallest path w.r.t. reverse dictionary order between $i$ and $j$. It is now enough to show that every edge in the path $P(i, j)$ satisfies the characterization given in Lemma 4. Suppose if possible, let $e \in P(i, j)$ be of smallest possible weight such that $e$ is incident on nodes in a same connected component of $\mathcal{G}_{<w(e)}$. Adding $e$ thus forms a cycle $C$ and the other edges in $C$ have weights strictly less than $w(e)$.

Now, consider the subgraph generated by edges in $P(i, j) \cup C \backslash\{e\}$. This subgraph is connected and hence there exists a path $Q(i, j)$ (say) between $i$ and $j$. It is easy to see that $Q(i, j)$ has smaller reverse dictionary order compared to $P(i, j)$ : number of edges of weight greater than $w(e)$ in $Q(i, j)$ cannot exceed to that in $P(i, j)$ since $C$ has edges of weight strictly less than $w(e)$; number of edges of weight $w(e)$ in $Q(i, j)$ is at least one less than that of $P(i, j)$. This contradicts the fact that $P(i, j)$ is a smallest path w.r.t. reverse dictionary order between $i$ and $j$.

Corollary 2 For every pair $i$ and $j$ in $\mathcal{G}$, we have $\eta(i, j)=\Delta(i, j)$.

The main result is formally stated as follows:
Theorem 1 As $p \rightarrow \infty$, we have the following:

$S P F_{i}^{(p)} \longrightarrow U M S T_{i}$

In other words, the shortest path filters converge to the UMST filter as $p \rightarrow \infty$.

Proof The proof follows readily from Proposition 2 and Lemma 1.

A key takeaway of the above result is one can exploit these links to build novel algorithms for edge-aware and attribute-specific filtering. For instance, the adaptive spanning trees (that we shall shortly introduce in Proposition 3 of Sec 5) can be used to learn features for supervised edge-aware filtering.

\section{Implementation}

In this section, we discuss several possible implementations of UMST filter. We utilize ideas from shortest paths and spanning trees to obtain two novel approximation algorithms to compute UMST filter. We provide detailed analyses of our implementations along with the TF which is yet another approximation of the UMST filter.

\subsection{Exact Algorithms}

In order to obtain the UMST filter, we shall first compute the UMST. As we work on color images and that we use $l_{1}$ norm (see Eq 7 ), the number of distinct edgeweights are practically constant. Using a bucket sort and the UMST characterization given by Lemma 4, one can obtain the UMST in $\mathcal{O}(|E|)$. Further, as the graph constructed from the image is a 4-adjacency graph, we have $\mathcal{O}(|V|)=\mathcal{O}(|E|)$ thus we can compute UMST in $\mathcal{O}(|V|)$.

A naive approach is to adapt the Floyd-Warshall [21] algorithm to calculate $\eta(i, j)$ for each pair and the computation of UMST filter takes $\mathcal{O}\left(|V|^{3}\right)$ time and $\mathcal{O}\left(|V|^{2}\right)$ space. This is very expensive in terms of both memory and space and is hence not practical. In order to reduce the complexity, one needs to exploit the properties of lexicographic ordering and that of the UMST graph structure. One approach is by borrowing ideas from Image Foresting Transform (IFT) [19,11].

Image Foresting Transform is a unified framework for several image processing operators that are based on shortest paths. Some of these operators include fuzzyconnected segmentation $[12,29]$ and distance transforms 
$[26,18]$. In simple words, IFT is a generalization of Dijkstra's algorithm where an image (with a specified adjacency relation), a set of seeds and a path cost function are specified and one needs to assign to every nonseeded pixel, a seed label to which it admits a path with smallest cost. The path costs are usually applicationspecific and are not necessarily given by sum of the weights of the edges on the path. Hence, a modified Dijkstra's algorithm is used to handle a general class of path cost functions that arise in computer vision applications. Although, sufficient conditions for a path cost were first proposed in [19], these are actually insufficient and have been corrected in [11]. We briefly describe the IFT framework below as in [19] and then discuss the relations with the SPF's:

The IFT takes as an input, an image $I$, an adjacency relation $E$ (usually given by 4 -adjacency in case of $2 \mathrm{D}$ images), a cost function $f$ for all paths and outputs an optimum spanning forest. Note that the seeds can be specified implicitly by the cost function by assigning a fixed cost for every path that starts at a certain pixel (finite for seed pixels and infinite for non-seed pixels). Although, there are no restrictions on the dimension of the image and the adjacency relation, there is a restriction on the path costs. Monotonic-incremental path costs form a special case of the most general conditions under which the IFT algorithm [19,11] yields correct results. These conditions suffice for our purposes and are described below:

The cost function $f$ is monotonic-incremental (MI) if it satisfies:

$-f(<t>)=h(t)$

$-f(P(i, s) .<s, t>)=f(P(i, s)) \odot\{s, t\}$

where $h(i)$ is an arbitrary but fixed cost, $P(i, s)$ is a path from $i$ to $s$ and $\odot: \mathbb{R}^{+} \times E \rightarrow \mathbb{R}^{+}$is a binary operation that satisfies the following conditions

$-x^{\prime} \geq x \Rightarrow x^{\prime} \odot\{s, t\} \geq x \odot\{s, t\}$,

$-x \odot\{s, t\} \geq x$,

for any $x, x^{\prime} \in \mathbb{R}^{+}$and $\{s, t\} \in E$.

Using a single seed, the optimum-path forest obtained (which is a tree rooted at the seed) can be used to compute the shortest path filtering collaborative weights (see Eq 6). By varying the seed $s$, the SPF can be computed for the whole image. However, such an implementation would still take $\mathcal{O}\left(|V|^{2}\right)$ time. To the best of our knowledge, we do not have any linear-time exact algorithms for computing the UMST filter. Thus, it calls a need to develop at least a quasi-linear algorithm approximation algorithm.

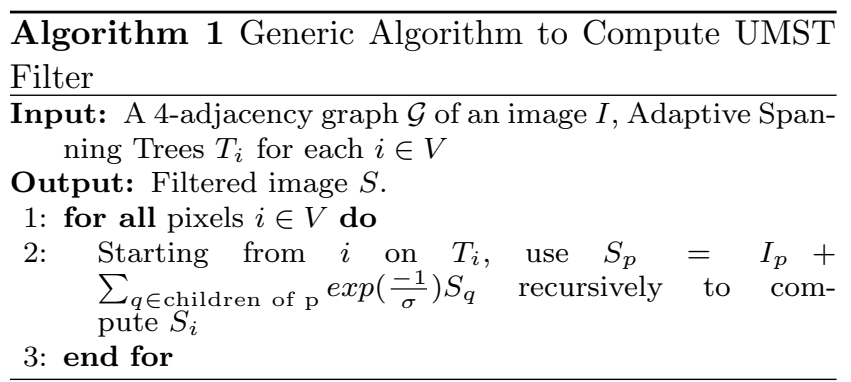

\subsection{Approximation Algorithms}

Single tree-based approximation: As we have developed the UMST filter by generalizing the notion of TF, we can view TF as a heuristic approximation of the UMST filter. Note that TF uses only one MST and hence can be computed dynamically in linear time by doing an upward aggregation followed by a downward aggregation on the tree (see [40] for details). However, the usage of an arbitrary MST makes it difficult to analyse the degree of approximation quantitatively.

Multiple tree-based approximations: One can also uses multiple spanning trees adaptively for filtering different pixels. In fact, one can find upper bounds on the approximation factors as a consequence of Proposition 3.

Proposition 3 For every pixel $i$ in the image I, there exists a spanning tree $T_{i}$ (termed as adaptive spanning tree), such that $T_{i}$ contains a smallest path with respect to reverse dictionary ordering between pixels $i$ and any other pixel $j$ in $I$.

Proof Let $i$ be an arbitrary pixel in $I$. We shall construct an adaptive spanning tree $T_{i}$, such that $T_{i}$ contains a smallest path with respect to reverse dictionary ordering between pixels $i$ and any other pixel $j$ in $I$. The construction is a special case of IFT (see [19]) with the following as the inputs: $I$ is the image with 4-adjacency. The path costs are given by: $f(P)=\infty$ for every path $P$ that does not start at $i$. All paths $P_{l}$ that start at $i$ are completely ordered in the order of decreasing reverse lexicographic ordering using their edgeweight distributions. The path costs are determined by the order statistics of the path i.e. if $P_{1} \geq P_{2} \geq \cdots>P_{l}$ are all the paths starting from $i$ ordered w.r.t. reverse lexicographic ordering then the path cost $f$ is given by $f\left(P_{t}\right)=l-t+1$ where $1 \leq t \leq l$. We remark that the this path cost is monotonic incremental (see [19,11]). The IFT algorithm applied thus yields $T_{i}$, an adaptive spanning tree of $i$ with the required properties.

Proposition 3 essentially implies that one can decompose the UMST into possibly different spanning 


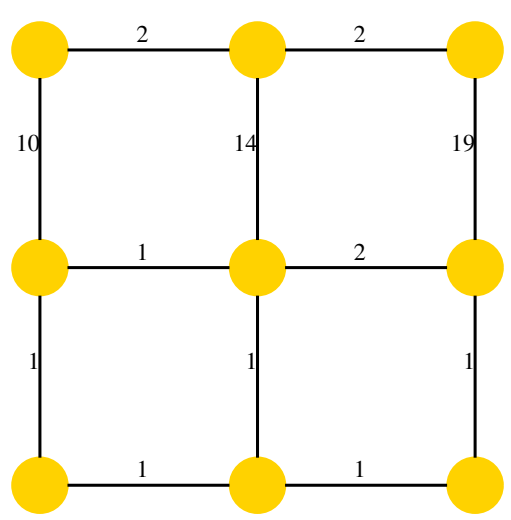

(a)

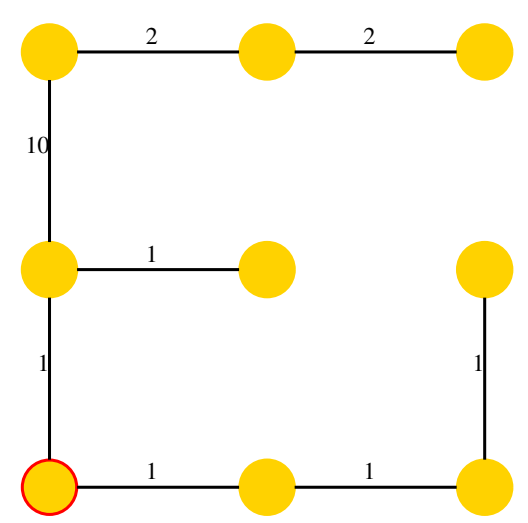

(b)

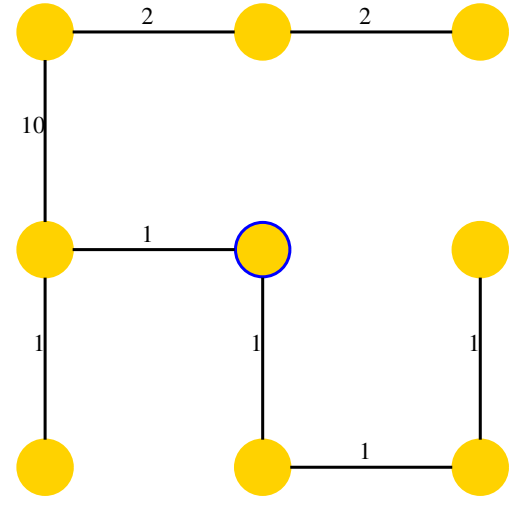

(c)

Fig. 5 (a) A synthetic image with the edge-weights reflecting the intensity dissimilarities, (b) and (c) Adaptive spanning trees of the pixels circled in red and blue respectively.

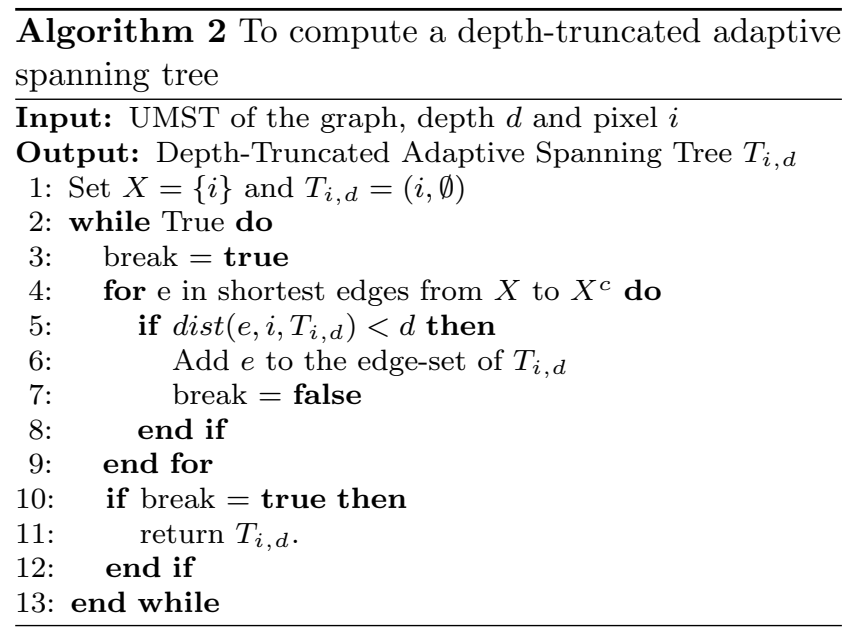

trees $T_{i}$ for each $i \in V$. Using each of the trees independently (see Fig 5 for an illustration on a synthetic image), one can obtain the exact UMST filter (see Algorithm 1). The exact computation takes $\mathcal{O}\left(|V|^{2}\right)$ and $\mathcal{O}(|V|)$ time and space complexities respectively. However, by truncating each of these trees in Algorithm 1, one can obtain fast approximate solutions. We present two ways to truncate the adaptive trees to obtain approximate UMST filter.

Depth-based truncation: For each $i \in V$, we truncate the adaptive spanning tree $T_{i}$ to $T_{i, d}$ such that it contains only the pixels $j$ that are at most $d$ (user-defined parameter) edges away from $i$ on $T_{i}$ (see Fig 6(b) for an illustration and Algorithm 2 for computing it)

We rewrite Eq 19 as:

$U M S T_{i}=\frac{1}{C} \sum_{l} \exp \left(-\frac{l}{\sigma}\right) \sum_{j: \eta(i, j)=l} I_{j}$, where $C$ is the normalizing constant $\sum_{l} \exp \left(-\frac{l}{\sigma}\right)$. In Eq 21 , we observe that the exponential term rapidly converges to 0 and hence one can approximate the above expression by

$U M S T_{i} \approx U M S T_{i, d}=\frac{1}{C^{\prime}} \sum_{l=1}^{d} \exp \left(-\frac{l}{\sigma}\right) \sum_{j: \eta(i, j)=l} I_{j}$,

where $d$ is a parameter indicating a fixed depth and $C^{\prime}$ is equal to $\sum_{l=1}^{d} \exp \left(-\frac{l}{\sigma}\right) \sum_{j: \eta(i, j)=l} 1$. This simplification reduces the calculation for each pixel drastically and hence Algorithm 2 is practically $\mathcal{O}(|V|)$.

We shall now analyse Eq 22 in more detail. Also as a consequence of Proposition 3, any two pixels are separated by at most $|V|-1$ edges on a spanning tree $T_{i}$ i.e. $\eta(i, j) \leq|V|-1$. Also, if $\eta(i, j)=l>0$ then for each $0 \leq l^{\prime} \leq l$, there exists at least one pixel $j^{\prime}$ such that $\eta\left(i, j^{\prime}\right)=l^{\prime}$. Assume that the intensities satisfy $1 \leq I_{j} \leq 255$ then we have the following (here we denote $U M S T$ by $U$ and $U M S T_{i}$ by $U_{i}$ to simplify the notation):

$$
\begin{array}{r}
\frac{U_{i}-U_{i, d}}{U_{i}}=\frac{\sum_{l=d+1}^{|V|-1} \exp \left(-\frac{l}{\sigma}\right) \sum_{j: \eta(i, j)=l} I_{j}}{\sum_{l=0}^{|V|-1} \exp \left(-\frac{l}{\sigma}\right) \sum_{j: \eta(i, j)=l} I_{j}} \\
\leq \frac{\exp \left(-\frac{d+1}{\sigma}\right) \sum_{j: \eta(i, j) \geq d+1} I_{j}}{I_{i}+\sum_{l=1}^{d} \exp \left(-\frac{l}{\sigma}\right) \sum_{j: \eta(i, j)=l} I_{j}} \\
\leq \frac{\exp \left(-\frac{d+1}{\sigma}\right) \sum_{j: \eta(i, j) \geq d+1} I_{j}}{1+\sum_{l=1}^{d} \exp \left(-\frac{l}{\sigma}\right)} \\
=\frac{\exp \left(-\frac{d+1}{\sigma}\right)}{1+\sum_{l=1}^{d} \exp \left(-\frac{l}{\sigma}\right)} \sum_{j: \eta(i, j) \geq d+1} I_{j} \\
=\frac{\exp \left(-\frac{d+1}{\sigma}\right)}{1-\exp \left(-\frac{d+1}{\sigma}\right)}\left(1-\exp \left(-\frac{1}{\sigma}\right)\right) \sum_{j: \eta(i, j) \geq d+1} I_{j}
\end{array}
$$




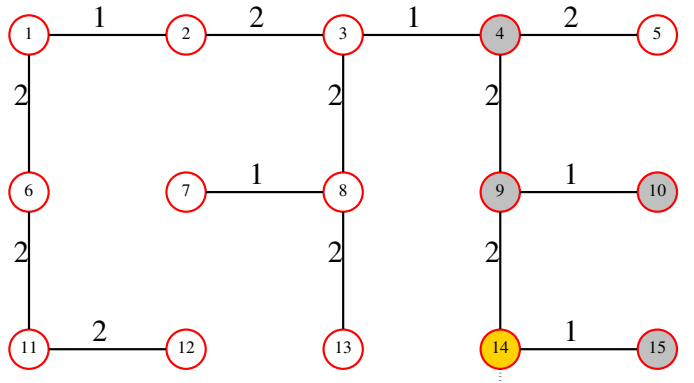

10

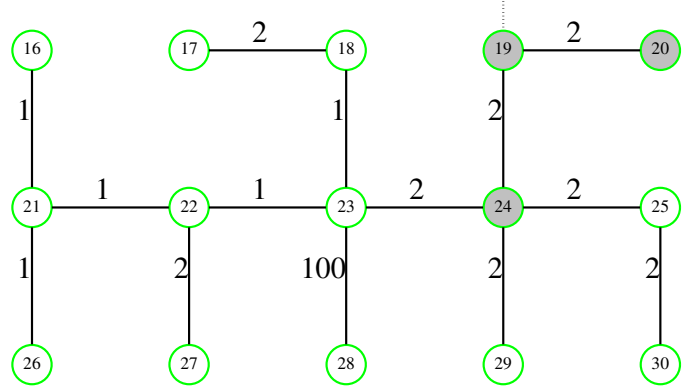

(a)

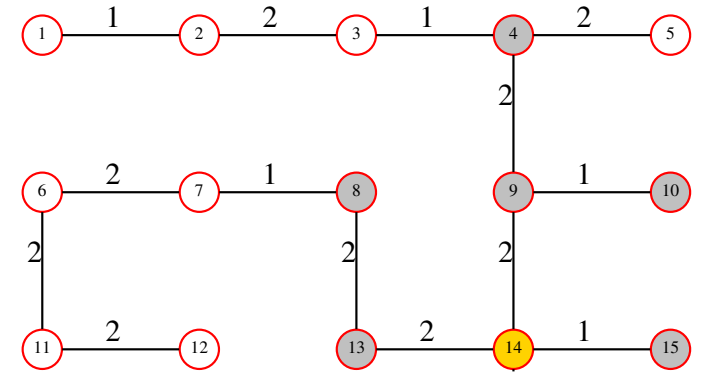

10 .

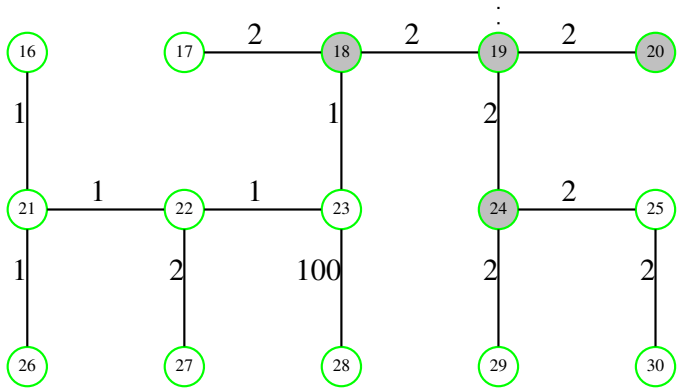

(b)

Fig. 6 (a) TF of pixel numbered 14 in Fig 2, here the pixels with significant collaboration (distance $\leq 2$ ) are highlighted in grey (b) Depth-based truncation of UMST filter at pixel numbered 14 in Fig 2, here the pixels with significant collaboration $($ depth $\leq 2)$ are highlighted in grey. Observe that the collaboration within the object is higher due to the usage of adaptive spanning tree instead of an arbitrary MST.

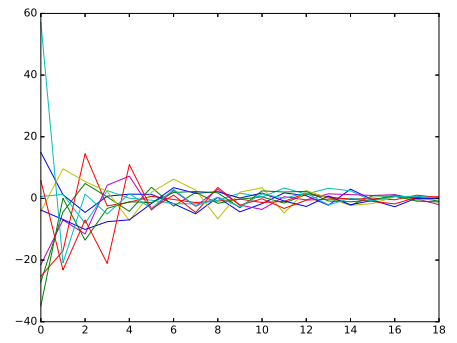

(a)

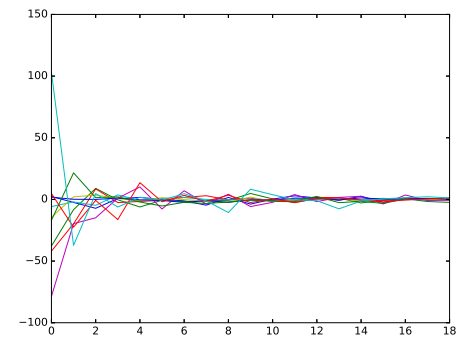

(b)

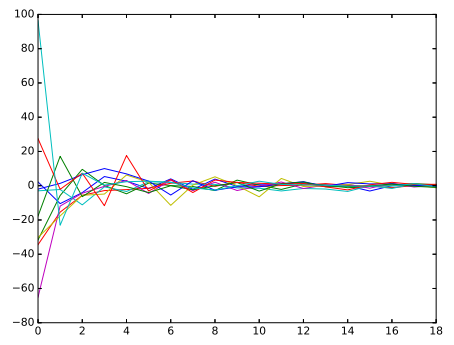

(c)

Fig. 7 From a color image, many pixels have been chosen randomly and each curve in a sub figure represents a pixel. The RGB bands are separately processed and plotted in three sub figures. In each of the sub figures, a curve denotes the first difference of the depth-truncated approximate UMST filtered values as a function of depth. Note that the differences stabilize to 0 at a depth of 15 indicating that Eq 22 yields good approximation to UMST filter.

For an image with $10^{6}$ pixels, setting $\sigma=0.1$, the expression in Eq 27 is bounded above by $\frac{1}{100}$ whenever $d \geq 220$. However, the empirical results illustrated by Fig 7 indicate that the filtered value of the pixel as a function of depth, $d$, stabilizes beyond a depth of 15 for $\sigma=0.1$.

In what follows, we view a rooted spanning tree $T$ as a directed spanning tree: for every pixel $j$, a path $P^{*}(j)$ recursively as $\langle j\rangle$ if parent of $j$ i.e. $\operatorname{Parent}(j)=$ null, and $P^{*}(j)=P^{*}(s) \cdot\langle s, j\rangle$ if Parent $(j)=s \neq$ null (notations are borrowed from [19]).
Order-based truncation: For each $i \in V$, we truncate the adaptive spanning tree $T_{i}$ to $\hat{T}_{i, N}$ such that it contains only the pixels $j$ among the closest $N$ (userdefined parameter) pixels w.r.t. the lexicographic ordering from $i$ on $T_{i}$. (see Fig 8(b) for an illustration and Algorithm 3 for computing it)

The order-based truncation of the adaptive spanning tree would precisely compute the limit of the morphological amoeba filters with $\lambda=1$ in Eq 10. More formally, we have the following result:

Proposition 4 As $p \rightarrow \infty$, we have 


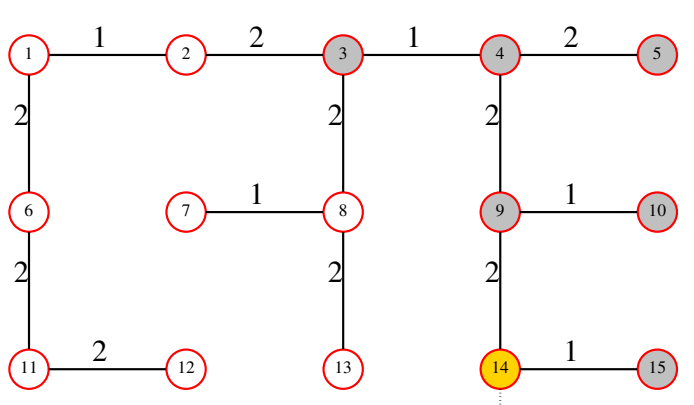

10

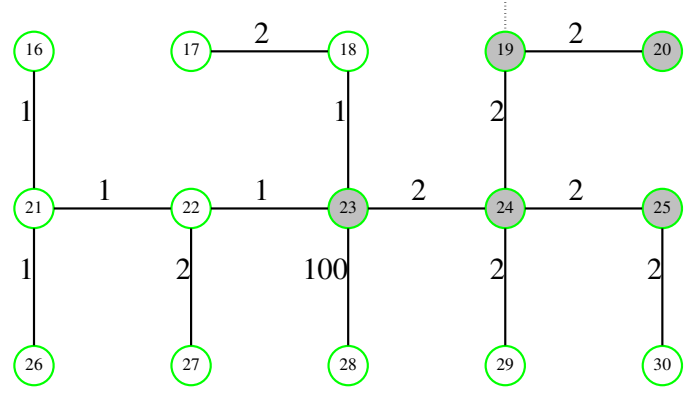

(a)

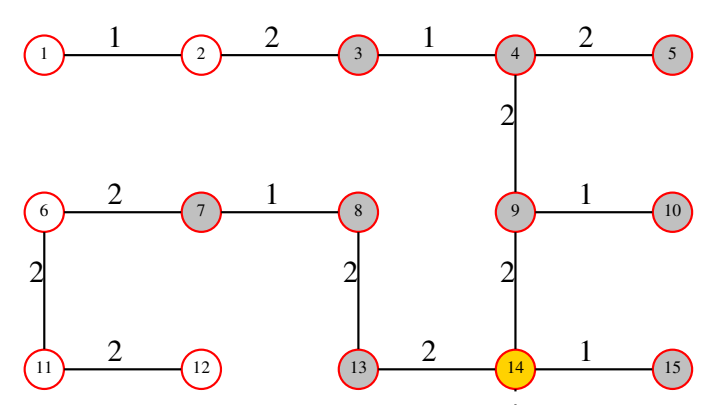

10.

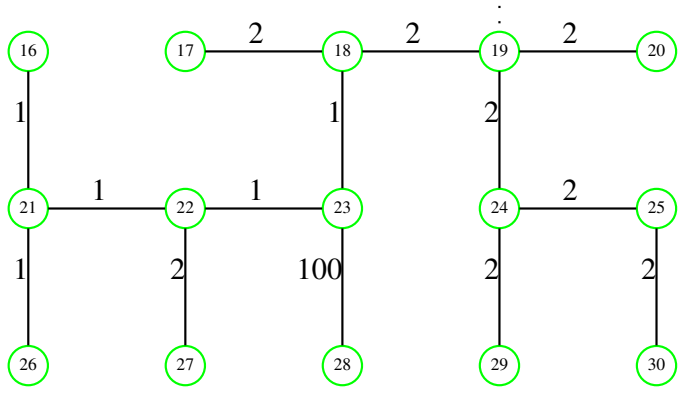

(b)

Fig. 8 (a) TF of pixel numbered 14 in Fig 2, here the pixels that contribute the highest to pixel numbered 14 (top 12 including itself) are highlighted in grey (b) Order-based truncation of PTF at pixel numbered 14 in Fig 2 , here the pixels that are closest w.r.t. lexicographic order from 14 (top 12 including itself) are highlighted in grey. Observe that the collaboration in the PTF within the object is very high due to the usage of adaptive spanning tree instead of an arbitrary MST.

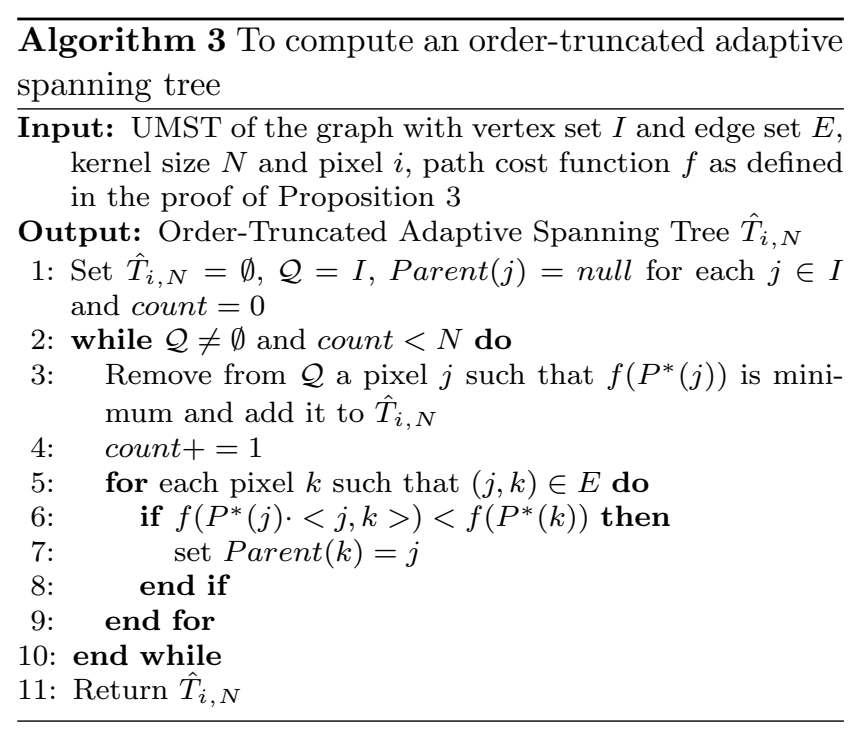

$S P F_{i, N}^{(p)} \longrightarrow U \hat{M} S T_{i, N}$

where $S P F_{i, N}^{(p)}$ is the morphological amoeba filter at pixel $i$ (with $\lambda=1$ and kernel size $N$ ) and $U \hat{M} S T_{i, N}$ $i s$ the collaborative filter using the closest $N$ pixels to $i$ w.r.t. the reverse lexicographic ordering on the adaptive spanning tree $T_{i}$.

\section{Proof The proof follows directly from Lemma 1.}

We remark that the choice of the kernel size $N$ determines the trade-off between the level of smoothing and the computational cost. In practice, using $N \approx 100$, one can obtain a good edge-aware filter. As the kernel size is fixed and small, Algorithm 3 runs practically in $\mathcal{O}(|V|)$ time. We shall see the comparison of the performance of our approximations with that of tree filter in the experiments section.

\section{Experiments}

In this section, we demonstrate that our approximations of the UMST filter perform similar to the TF (in fact marginally better) at an additional computational cost. To process 1000 pixels, it takes about $0.29 \mathrm{sec}-$ onds, 37 seconds and 738 seconds for TF, order-based and depth-based approximations respectively. Consequently, we provide an empirical evidence that $\mathrm{TF}$ is a fast approximation to the limit of the SPFs. For all our experiments, we have used identical $\sigma(=10)$ parameter for computing the TF, depth-based (with depth $=15$ ) and order-based (with $N=100$ ) multi-tree approximations of UMSTF. The experiments are performed on Intel (R) Xeon(R) CPU W3565 at $3.20 \mathrm{GHz}$ with RAM size of 6 GigaBytes. 


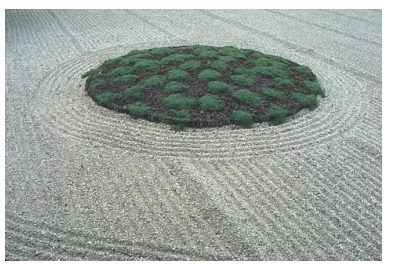

(a)

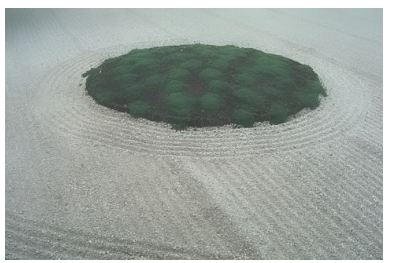

(e)

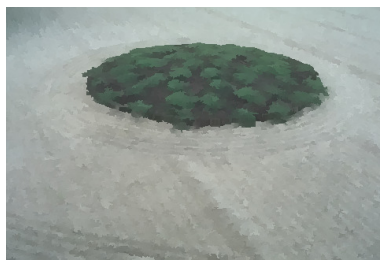

(i)

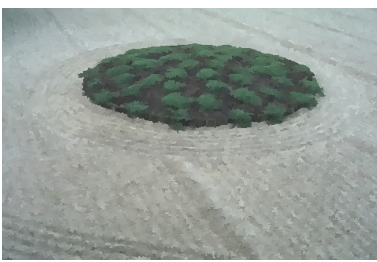

(m)

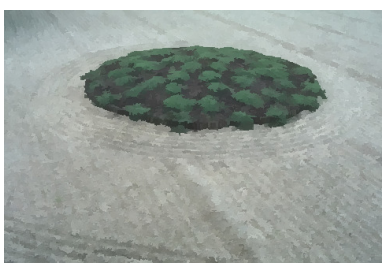

(q)

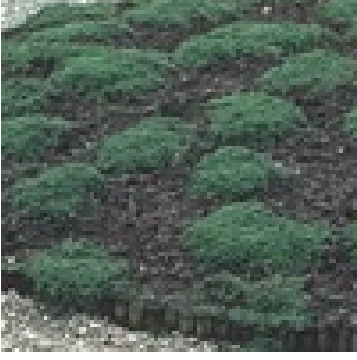

(b)

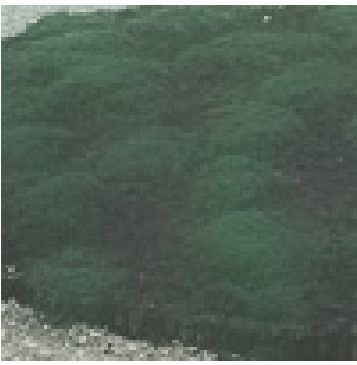

(f)

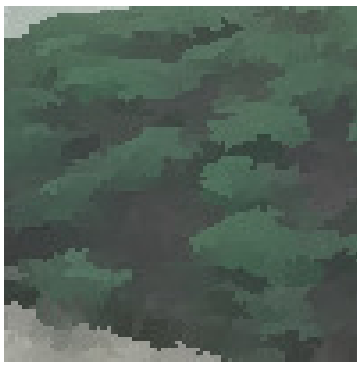

(j)

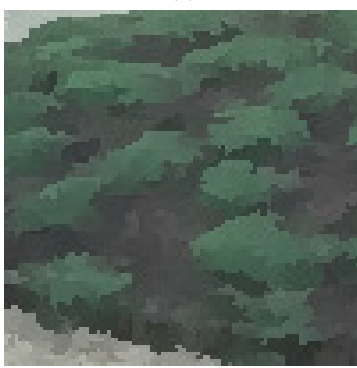

(n)

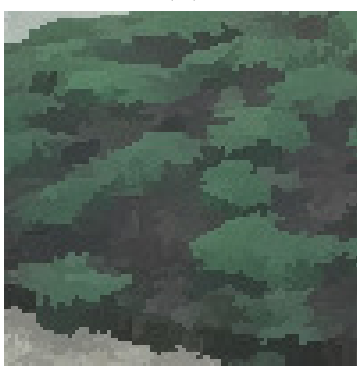

(r)

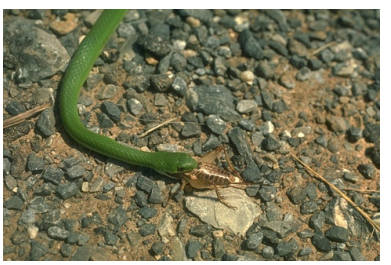

(c)

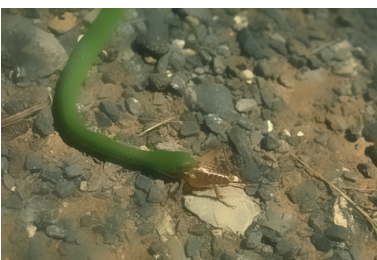

(g)

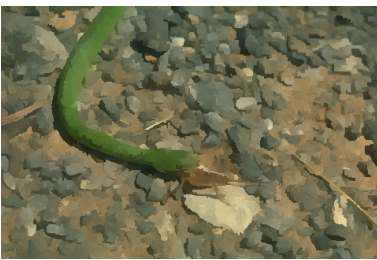

(k)

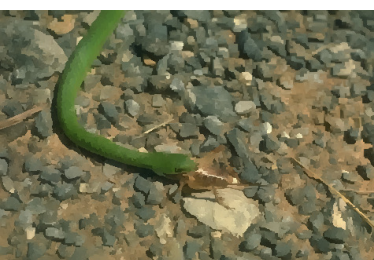

(o)

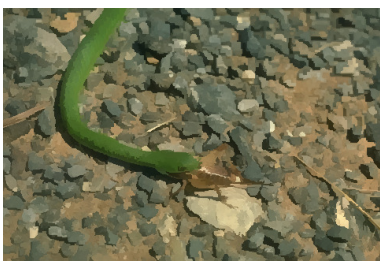

(s)

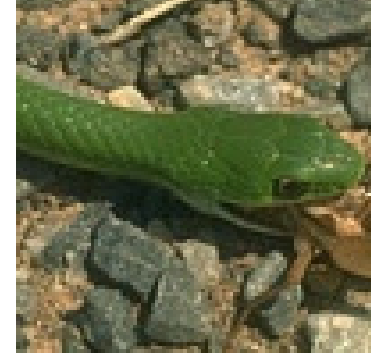

(d)

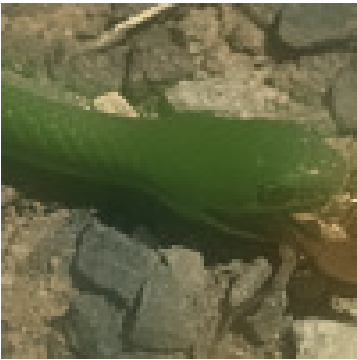

(h)

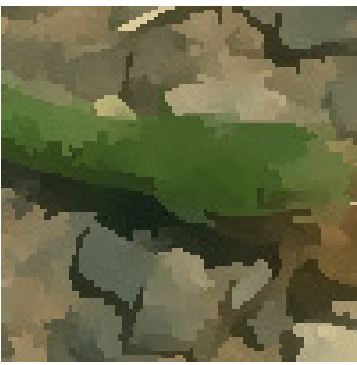

(1)

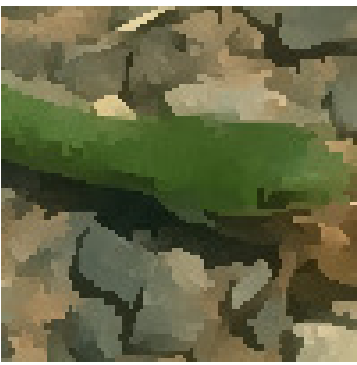

(p)

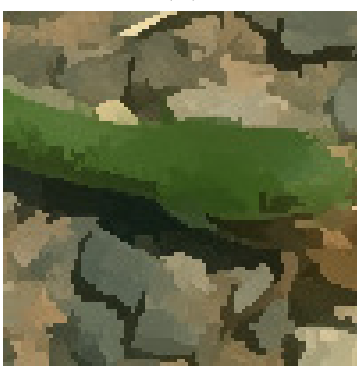

(t)

Fig. 9 A visual comparison of the subtle differences in the performance of TF and our multi-tree approximations of UMSTF on BSDS500 [3] images are illustrated. (a), (b), (c), (d) original images (e), (f), (g), (h) Bilateral Filter $(\sigma$ color = 100, $\sigma$ space $=10)(\mathrm{i}),(\mathrm{j}),(\mathrm{k}),(\mathrm{l})$ Tree Filter $(\sigma=10)(\mathrm{m}),(\mathrm{n}),(\mathrm{o}),(\mathrm{p})$ Depth-based multi-tree truncation $(\sigma=10$, depth $=15)$ (q), (r), (s), (t) Order-based multi-tree truncation $(\sigma=10, N=100)$. In the second and fourth columns, observe the green patches and the snake's eye respectively. The leaks are more prominent in TF when compared to our approximations. These comparisons are meant for a qualitative comparison only. 
Firstly, Fig 9 shows a qualitative comparison of BF, $\mathrm{TF}$, depth-based and order-based multi-tree approximations on some natural images. We observe that $\mathrm{BF}$ yields blurry images and erases some object boundaries as expected. On the other hand, TF and our approximations yield similar results. However, on a closer look, one can observe that the boundaries are marginally better preserved in our approximations (compare green patches in Fig 9 second column, snake's eye in Fig 9 fourth column).

For a quantitative comparison of these filters, we have computed the PSNR and structural similarity index (SSIM) [8] on images corrupted with synthetic noises. In general, higher PSNR values and higher SSIM (SSIM equal to 1 implies identical structures) indicate that the image structures are better preserved. However, SSIM is a superior measure when compared to PSNR as the latter estimates absolute errors while the former takes structural information into consideration. To see this, observe that the mean PSNR values of BF in Table 1 is higher than that of other two filters over three iterations of random salt and pepper noise. However, a visual comparison of these filters (see Fig 10) suggests that noise is better eliminated by $\mathrm{TF}$ and our approximation. The mean SSIM values however (see Table 2) are in-line with the visual results and indicate that orderbased approximation of UMST filter performs better than TF in presence of salt and pepper noise. Further, the scatter plot (see Fig 11) of the SSIM values of TF and order-based approximation of UMST filter on three iterations of random Poisson, salt and pepper, Gaussian and speckle noises on these images (House, Barbara, Lena and Pepper) indicate that our approximation is slightly better than TF irrespective of the type of noise.

Table 1 Peak Signal to Noise Ratios (PSNR) measured in $\mathrm{db}$ on filtered images corrupted by salt and pepper noise. A higher value indicates a better filter. Here BF, TF and PTF indicate bilateral filter, tree filter and order-based truncation of UMST filter respectively.

\begin{tabular}{|l|c|c|c|}
\hline & Mean PSNR on Salt Pepper Noise \\
\hline & BF & TF & PTF \\
\hline House & 24.71 & 25.04 & 24.67 \\
\hline Barbara & 24.08 & 22.19 & 22.47 \\
\hline Lena & 22.58 & 22.59 & 22.64 \\
\hline Pepper & 21.70 & 21.96 & 20.59 \\
\hline Mean & $\mathbf{2 3 . 2 7}$ & $\mathbf{2 2 . 9 5}$ & $\mathbf{2 2 . 5 9}$ \\
\hline
\end{tabular}

Although our approximations yield marginally better results than that of $\mathrm{TF}$, they are computationally expensive. However, it is important to note that our approximations can be implemented in parallel as each pixel is processed independently of the other. Also we note that one has to choose an appropriate filter de-
Table 2 Structural Similarity Indices (SSIM) on filtered images corrupted by salt and pepper noise. A higher value indicates a better filter and a value close to 1 indicates an ideal filter. Here $\mathrm{BF}, \mathrm{TF}$ and $\mathrm{PTF}$ indicate bilateral filter, tree filter and order-based truncation of UMST filter respectively.

\begin{tabular}{|l|c|c|r|}
\hline & \multicolumn{3}{|c|}{ Mean SSIM on Salt Pepper Noise } \\
\hline & BF & TF & PTF \\
\hline House & 0.69 & 0.80 & 0.83 \\
\hline Barbara & 0.72 & 0.66 & 0.72 \\
\hline Lena & 0.69 & 0.75 & 0.79 \\
\hline Pepper & 0.62 & 0.74 & 0.74 \\
\hline Mean & $\mathbf{0 . 6 8}$ & $\mathbf{0 . 7 4}$ & $\mathbf{0 . 7 7}$ \\
\hline
\end{tabular}

pending on the type of noise. For instance, BF outperforms the tree-based filters in presence of Gaussian noise (see Table 3 and Table 4). To summarize, we have demonstrated that $\mathrm{TF}$ is a fast approximation of the limit of SPFs.

Table 3 Peak Signal to Noise Ratios (PSNR) measured in $\mathrm{db}$ on filtered images corrupted by Gaussian noise. A higher value indicates a better filter. Here $\mathrm{BF}, \mathrm{TF}$ and $\mathrm{PTF}$ indicate bilateral filter, tree filter and order-based truncation of UMST filter respectively.

\begin{tabular}{|l|c|c|c|}
\hline & \multicolumn{3}{|c|}{ Mean PSNR on Gaussian Noise } \\
\hline & BF & TF & PTF \\
\hline House & 25.12 & 23.76 & 23.97 \\
\hline Barbara & 24.14 & 21.18 & 21.58 \\
\hline Lena & 22.67 & 21.47 & 21.78 \\
\hline Pepper & 22.15 & 21.09 & 20.93 \\
\hline Mean & $\mathbf{2 3 . 5 2}$ & $\mathbf{2 1 . 8 8}$ & $\mathbf{2 2 . 0 7}$ \\
\hline
\end{tabular}

Table 4 Structural Similarity Indices (SSIM) on filtered images corrupted by Gaussian noise. A higher value indicates a better filter and a value close to 1 indicates an ideal filter. Here BF, TF and PTF indicate bilateral filter, tree filter and order-based truncation of UMST filter respectively.

\begin{tabular}{|l|c|c|r|}
\hline & \multicolumn{3}{|c|}{ Mean SSIM on Gaussian Noise } \\
\hline & BF & TF & PTF \\
\hline House & 0.79 & 0.73 & 0.75 \\
\hline Barbara & 0.77 & 0.57 & 0.62 \\
\hline Lena & 0.76 & 0.66 & 0.69 \\
\hline Pepper & 0.75 & 0.67 & 0.68 \\
\hline Mean & $\mathbf{0 . 7 7}$ & $\mathbf{0 . 6 6}$ & $\mathbf{0 . 6 9}$ \\
\hline
\end{tabular}

\section{Conclusions}

In this paper, we have analysed the edge-aware filters from scratch by developing shortest path filters as a natural extension of Gaussian-like filters. Using the power watershed framework, we have provided a common optimization set-up for the well-known filters based on 


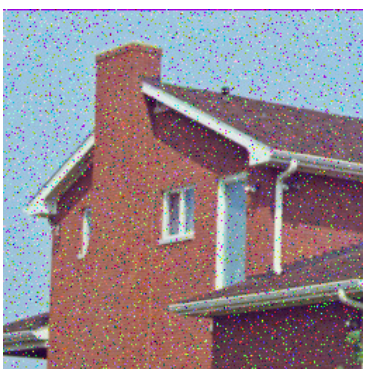

(a)

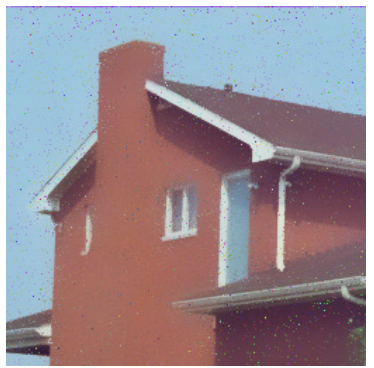

(e)

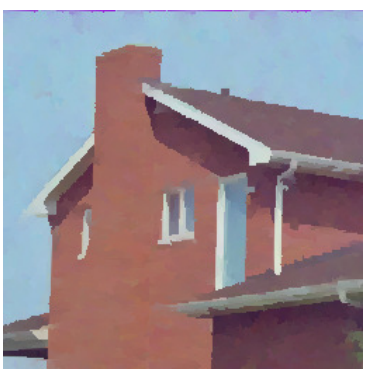

(i)

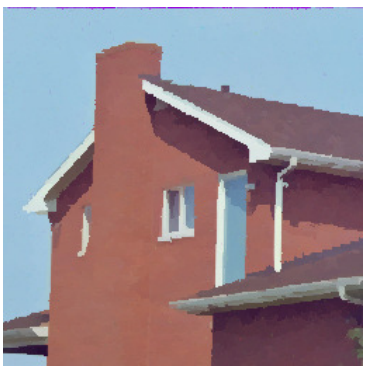

$(\mathrm{m})$

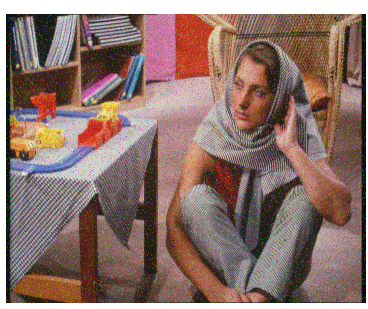

(b)

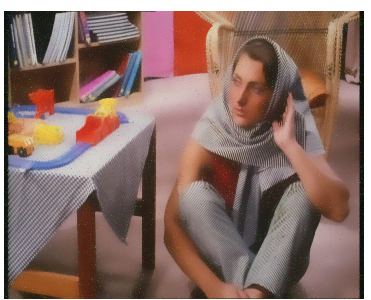

(f)

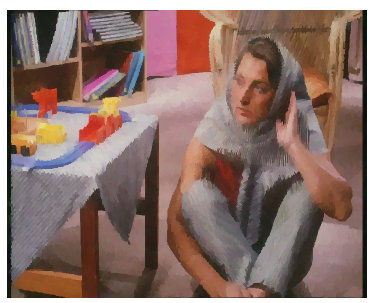

(j)

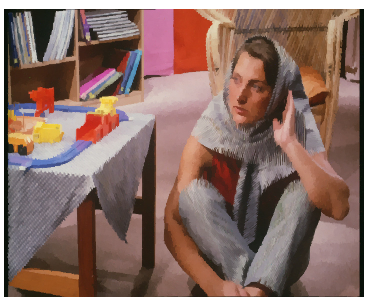

(n)

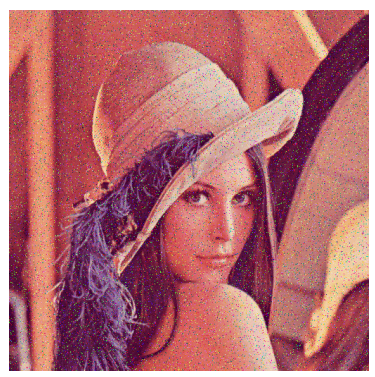

(c)

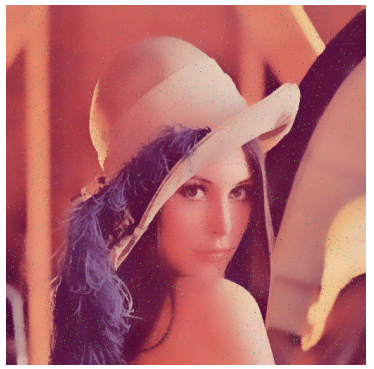

(g)

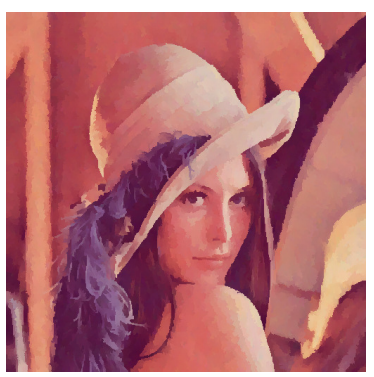

$(\mathrm{k})$

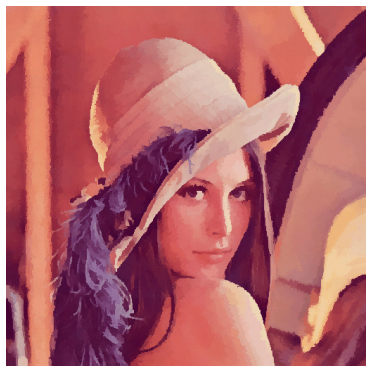

(o)

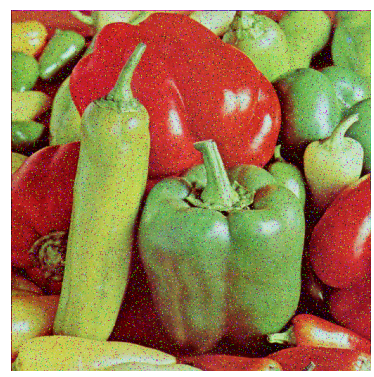

(d)

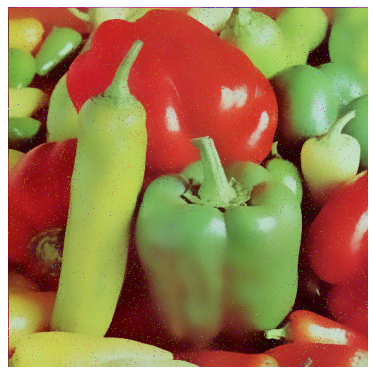

(h)

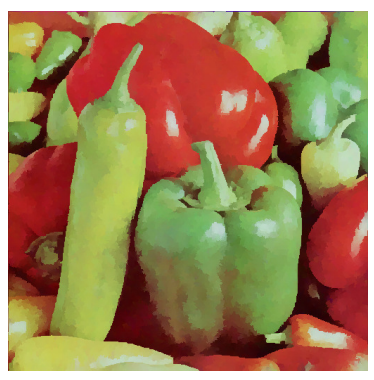

(l)

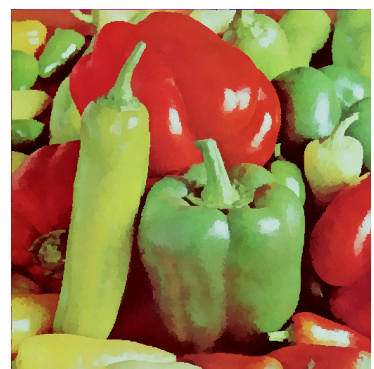

(p)

Fig. 10 Visual illustration of edge-preserving filters on images contaminated with salt and pepper noise. BF erases some object boundaries and does not eliminate the noise completely while TF and order-based approximation eliminate noise and yield similar results. (a), (b), (c), (d) Salt and pepper noisy images (e), (f), (g), (h) Bilateral Filter ( $\sigma$ color $=100, \sigma$ space = 10) (i), (j), (k), (l) Tree Filter $(\sigma=10)(\mathrm{m}),(\mathrm{n}),(\mathrm{o}),(\mathrm{p})$ Order-based multi-tree truncation $(\sigma=10, N=100)$

shortest paths and the ones based on minimum spanning trees. We have thus established a link to the tree filter by showing that it can be visualized as an approximate limit of shortest path filters. Further, we have proposed two different approximation algorithms of the UMST filter by leveraging ideas from shortest paths and minimum spanning trees.
Establishing methods based on principles and/or heuristics as limits of solutions of optimization problems would enable to design novel algorithms. For example, based on these theoretical foundations one may use the UMST adaptive spanning trees introduced in this paper to perform attribute filtering on tree of shapes 


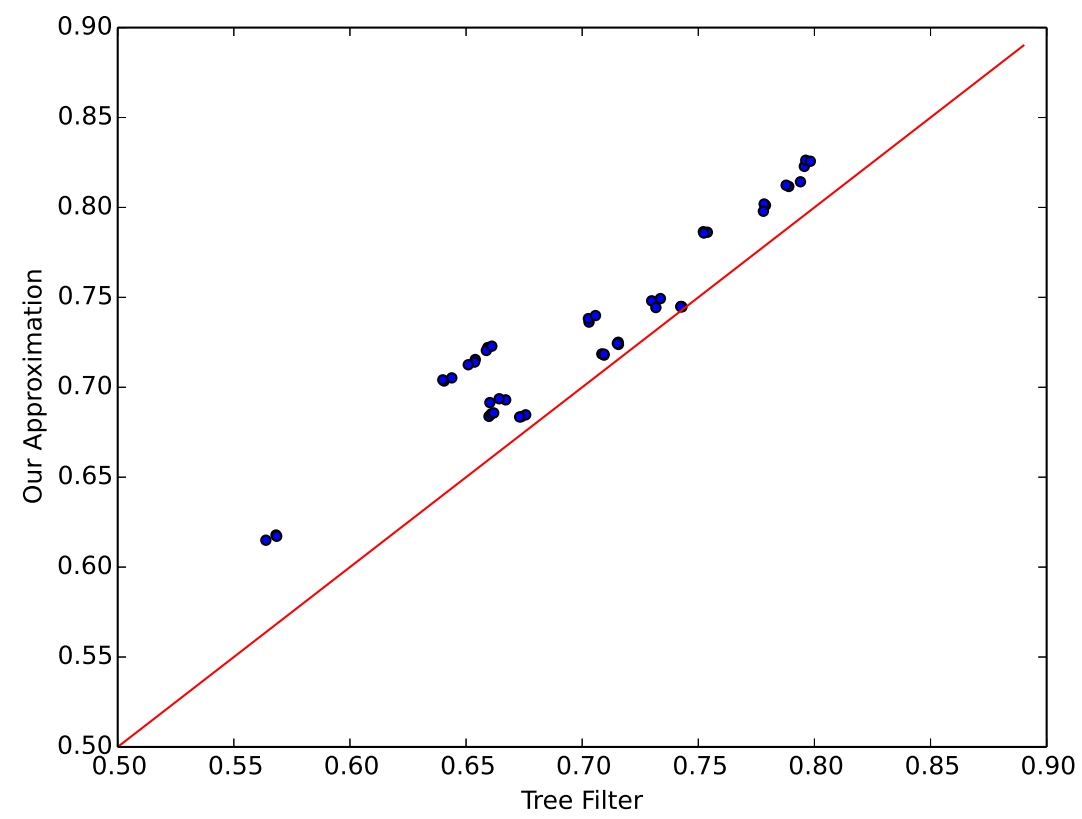

Fig. 11 Scatter plot of SSIM values of TF versus Order-based approximation of UMST filter on three iterations of random Poisson, Salt and Pepper, Gaussian and Speckle noises on House, Barbara, Lena and Pepper images.

[39] i.e. preserving specific shapes of interest while getting rid of the rest.

We believe that faster implementations can be developed using efficient GPU-based parallel algorithms [35] based on the ideas from our paper. Further, we believe that accuracy can be improved with supervised techniques by learning edge-aware features from the UMST adaptive spanning trees.

Ultimately, we aim to show that PW framework serves as a powerful tool in applications beyond image segmentation and filtering.

\section{Acknowledgments}

$\mathrm{SD}$ and $\mathrm{AC}$ would like to thank Indian Statistical Institute for providing fellowship to pursue the research. BSDS would like to acknowledge the funding received from EMR/2015/000853 SERB and ISRO/SSPO/Ch1/2016-17 ISRO research grants. LN would like acknowledge the funding received from Programme d'Investis sements d'Avenir (LabEx BEZOUT ANR-10-LABX58), ANR-15-CE40-0006 CoMeDiC and ANR-14-CE270001 GRAPHSIP research grants.

\section{References}

1. Alvino, C., Unal, G., Slabaugh, G., Peny, B., Fang, T.: Efficient segmentation based on eikonal and diffusion equa- tions. International Journal of Computer Mathematics 84(9), 1309-1324 (2007)

2. Angulo, J.: Pseudo-morphological image diffusion using the counter-harmonic paradigm. In: Advanced Concepts for Intelligent Vision Systems, pp. 426-437. Springer (2010)

3. Arbelaez, P., Maire, M., Fowlkes, C., Malik, J.: Contour detection and hierarchical image segmentation. IEEE transactions on pattern analysis and machine intelligence 33(5), 898-916 (2011)

4. Bai, X., Sapiro, G.: A geodesic framework for fast interactive image and video segmentation and matting. In: Computer Vision, 2007. ICCV 2007. IEEE 11th International Conference on, pp. 1-8. IEEE (2007)

5. Bao, L., Song, Y., Yang, Q., Yuan, H., Wang, G.: Tree filtering: Efficient structure-preserving smoothing with a minimum spanning tree. IEEE TIP 23(2), 555-569 (2014)

6. Boykov, Y.Y., Jolly, M.P.: Interactive graph cuts for optimal boundary \& region segmentation of objects in nd images. In: Computer Vision, 2001. ICCV 2001. Proceedings. Eighth IEEE International Conference on, vol. 1, pp. 105-112. IEEE (2001)

7. Braides, A.: Gamma-convergence for Beginners, vol. 22. Clarendon Press (2002)

8. Brunet, D., Vrscay, E.R., Wang, Z.: On the mathematical properties of the structural similarity index. IEEE Transactions on Image Processing 21(4), 1488-1499 (2012)

9. Challa, A., Danda, S., Daya Sagar, B.S., Najman, L.: An Introduction to Gamma-Convergence for Spectral Clustering. In: Discrete Geometry for Computer Imagery, Lecture Note In Computer Sciences, vol. 10502, pp. 185196. Kropatsch, Walter G. and Artner, Nicole M. and Janusch, Ines, Springer, Vienna, Austria (2017). URL https://hal.archives-ouvertes.fr/hal-01427957 
10. Chang, J.H.R., Wang, Y.C.F.: Propagated image filtering. In: 2015 IEEE Conference on Computer Vision and Pattern Recognition (CVPR), pp. 10-18. IEEE (2015)

11. Ciesielski, K.C., Falcão, A.X., Miranda, P.A.V.: Pathvalue functions for which dijkstra's algorithm returns optimal mapping. Journal of Mathematical Imaging and Vision (2018). DOI 10.1007/s10851-018-0793-1. URL https://doi.org/10.1007/s10851-018-0793-1

12. Ciesielski, K.C., Udupa, J.K., Falcão, A.X., Miranda, P.A.: Fuzzy connectedness image segmentation in graph cut formulation: A linear-time algorithm and a comparative analysis. Journal of Mathematical Imaging and Vision 44(3), 375-398 (2012)

13. Couprie, C., Grady, L., Najman, L., Talbot, H.: Power watershed: A unifying graph-based optimization framework. IEEE PAMI 33(7), 1384-1399 (2011)

14. Cousty, J., Bertrand, G., Najman, L., Couprie, M.: Watershed cuts: Minimum spanning forests and the drop of water principle. IEEE PAMI 31(8), 1362-1374 (2009)

15. Cousty, J., Bertrand, G., Najman, L., Couprie, M.: Watershed cuts: Thinnings, shortest path forests, and topological watersheds. IEEE PAMI 32(5), 925-939 (2010)

16. Criminisi, A., Sharp, T., Blake, A.: Geos: Geodesic image segmentation. Computer Vision-ECCV 2008 pp. 99-112 (2008)

17. Danda, S., Challa, A., Sagar, B.D., Najman, L.: Power tree filter: A theoretical framework linking shortest path filters and minimum spanning tree filters. In: International Symposium on Mathematical Morphology and Its Applications to Signal and Image Processing, pp. 199210. Springer (2017)

18. Falcão, A.X., da Fontoura Costa, L., Da Cunha, B.: Multiscale skeletons by image foresting transform and its application to neuromorphometry. Pattern recognition 35(7), 1571-1582 (2002)

19. Falcao, A.X., Stolfi, J., de Alencar Lotufo, R.: The image foresting transform: Theory, algorithms, and applications. IEEE PAMI 26(1), 19 (2004)

20. Farbman, Z., Fattal, R., Lischinski, D., Szeliski, R.: Edgepreserving decompositions for multi-scale tone and detail manipulation. In: ACM Transactions on Graphics (TOG), vol. 27 , p. $67 . \mathrm{ACM}(2008)$

21. Floyd, R.W.: Algorithm 97: shortest path. Communications of the ACM 5(6), 345 (1962)

22. Grady, L.: Random walks for image segmentation. IEEE PAMI 28(11), 1768-1783 (2006)

23. Grazzini, J., Soille, P.: Edge-preserving smoothing using a similarity measure in adaptive geodesic neighbourhoods. Pattern Recognition 42(10), 2306-2316 (2009)

24. He, K., Sun, J., Tang, X.: Guided image filtering. In: Computer Vision-ECCV 2010, pp. 1-14. Springer (2010)

25. Lerallut, R., Decencière, É., Meyer, F.: Image filtering using morphological amoebas. Image and Vision Computing 25(4), 395-404 (2007)

26. Lotufo, R.d.A., Falcão, A.A., Zampirolli, F.A.: Fast euclidean distance transform using a graph-search algorithm. In: Computer Graphics and Image Processing, 2000. Proceedings XIII Brazilian Symposium on, pp. 269-275. IEEE (2000)

27. Najman, L.: Extending the PowerWatershed framework thanks to $\Gamma$-convergence. SIAM Journal on Imaging Sciences 10(4), 2275-2292 (2017). DOI 10.1137/17M1118580. URL https://hal.archivesouvertes.fr/hal-01428875

28. Najman, L., Pesquet, J.C., Talbot, H.: When convex analysis meets mathematical morphology on graphs. In:
International Symposium on Mathematical Morphology and Its Applications to Signal and Image Processing, pp. 473-484. Springer (2015)

29. Saha, P.K., Udupa, J.K.: Relative fuzzy connectedness among multiple objects: theory, algorithms, and applications in image segmentation. Computer Vision and Image Understanding 82(1), 42-56 (2001)

30. Sinop, A.K., Grady, L.: A seeded image segmentation framework unifying graph cuts and random walker which yields a new algorithm. In: IEEE 11th International Conference on Computer Vision, 2007. ICCV 2007., pp. 1-8. IEEE (2007)

31. Stawiaski, J., Meyer, F.: Minimum spanning tree adaptive image filtering. In: 2009 16th IEEE ICIP, pp. 22452248. IEEE (2009)

32. Szeliski, R.: Computer vision: algorithms and applications. Springer Science \& Business Media (2010)

33. Tomasi, C., Manduchi, R.: Bilateral filtering for gray and color images. In: Sixth International Conference on Computer Vision, 1998. ICCV 1998, pp. 839-846. IEEE (1998)

34. Van Vliet, L.J.: Robust local max-min filters by normalized power-weighted filtering. In: Pattern Recognition, 2004. ICPR 2004. Proceedings of the 17th International Conference on, vol. 1, pp. 696-699. IEEE (2004)

35. Vineet, V., Harish, P., Patidar, S., Narayanan, P.: Fast minimum spanning tree for large graphs on the gpu. In: Proceedings of the Conference on High Performance Graphics 2009, pp. 167-171. ACM (2009)

36. Von Luxburg, U.: A tutorial on spectral clustering. Statistics and computing 17(4), 395-416 (2007)

37. Xu, L., Lu, C., Xu, Y., Jia, J.: Image smoothing via $L_{0}$ gradient minimization. In: ACM Transactions on Graphics (TOG), vol. 30, p. 174. ACM (2011)

38. Xu, L., Yan, Q., Xia, Y., Jia, J.: Structure extraction from texture via relative total variation. ACM Transactions on Graphics (TOG) 31(6), 139 (2012)

39. Xu, Y., Géraud, T., Najman, L.: Connected filtering on tree-based shape-spaces. IEEE transactions on pattern analysis and machine intelligence 38(6), 1126-1140 (2016)

40. Yang, Q.: Stereo matching using tree filtering. IEEE PAMI 37(4), 834-846 (2015)

41. Zahn, C.T.: Graph-theoretical methods for detecting and describing gestalt clusters. IEEE Transactions on computers 100(1), 68-86 (1971) 